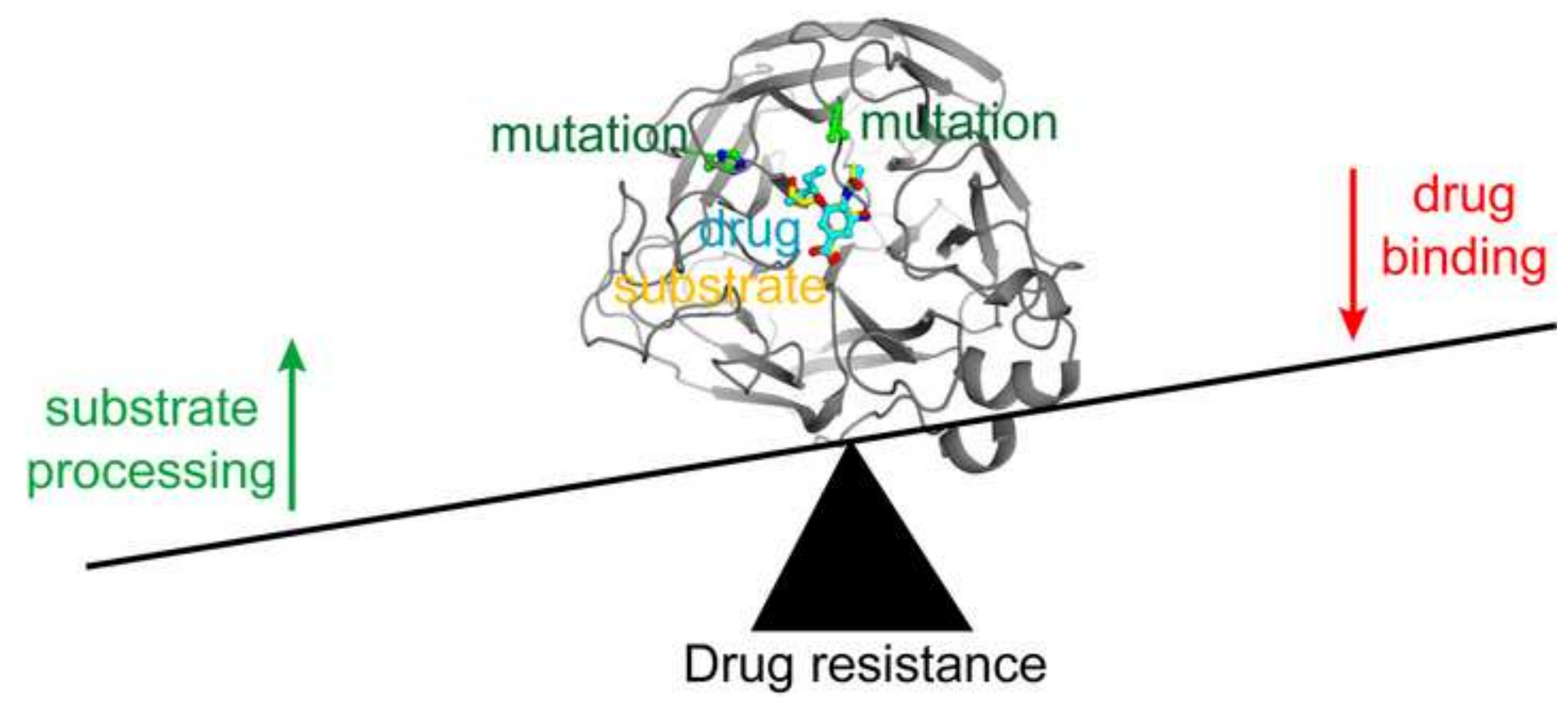




\title{
A balance between inhibitor binding and substrate processing confers influenza drug resistance
}

\author{
Li Jiang ${ }^{a}$, Ping Liu ${ }^{b}$, Claudia Bank $^{c}$, Nicholas Renzette ${ }^{d}$, Kristina Prachanronarong $^{a}$, Lutfu S. \\ Yilmaz $^{a}$, Daniel R. Caffrey ${ }^{b}$, Konstantin B. Zeldovich ${ }^{\mathrm{e}}$, Celia A. Schiffer ${ }^{\mathrm{a}}$, Timothy F. Kowalik ${ }^{\mathrm{d}}$, \\ Jeffrey D. Jensen ${ }^{c}$, Robert W. Finberg ${ }^{b}$, Jennifer P. Wang ${ }^{b, 1, \#, ~ D a n i e l ~ N . A . ~ B o l o n ~}{ }^{a, 1, \#}$ \\ ${ }^{\mathrm{a} D e p a r t m e n t ~ o f ~ B i o c h e m i s t r y ~ a n d ~ M o l e c u l a r ~ P h a r m a c o l o g y, ~ U n i v e r s i t y ~ o f ~ M a s s a c h u s e t t s ~}$ \\ Medical School, Worcester, MA, 01655, USA \\ ${ }^{b}$ Department of Medicine, University of Massachusetts Medical School, Worcester, MA, 01655, \\ USA \\ 'School of Life Sciences, École Polytechnique Fédérale de Lausanne (EPFL), 1015 Lausanne, \\ Switzerland \\ ${ }^{\mathrm{d} D e p a r t m e n t}$ of Microbiology and Physiological Systems, University of Massachusetts Medical \\ School, Worcester, MA, 01655, USA \\ ${ }^{\text {e}}$ Program in Bioinformatics and Integrative Biology, University of Massachusetts Medical \\ School, Worcester, MA, 01655, USA \\ ${ }^{1}$ J.P.W. and D.N.A.B. contributed equally to this work. \\ Email addresses of all authors: Li.Jiang@umassmed.edu, Ping.Liu@umassmed.edu, \\ Claudia.Bank@epfl.ch, Nicholas.Renzette@umassmed.edu, \\ Kristina.Prachanronarong@umassmed.edu, LutfuSSafak.Yilmaz@umassmed.edu, \\ Daniel.Caffrey@umassmed.edu,Konstantin.Zeldovich@umassmed.edu,
}


Celia.Schiffer@umassmed.edu,Timothy.Kowalik@umassmed.edu, Jeffrey.Jensen@epfl.ch, and

Robert.Finberg@umassmed.edu

" To whom correspondence should be addressed:

Jennifer.Wang@umassmed.edu

Ph: 1-508-856-8414

Fax: 1-508-856-6176

University of Massachusetts

364 Plantation Street, LRB 219

Worcester, MA 01605 or

Dan.Bolon@umassmed.edu

University of Massachusetts

364 Plantation Street, LRB 922

Worcester, MA 01605

Ph: 1-508-856-3588

Fax: 1-508-856-6464

Abbreviations: NA, neuraminidase; IAV, influenza A virus; HA, hemagglutinin; EMPIRIC,

Exceedingly Meticulous and Parallel Investigation of Randomized Individual Codons; MDCK,

Madin-Darby canine kidney, P0, initial viral pool; $\mathrm{IC}_{50}, 50 \%$ enzyme-inhibitory concentration; 
SLIC, sequence and ligation independent cloning; MEM, minimal essential medium; PFU, plaque forming units; IVGM, influenza virus growth medium; MOI, multiplicity of infection; MUNANA, 2'-(4-methylumbelliferyl)- alpha-D-N-acetylneuraminic acid 


\begin{abstract}
The therapeutic benefits of the neuraminidase (NA) inhibitor oseltamivir are dampened by the emergence of drug resistance mutations in influenza A virus (IAV). To investigate the mechanistic features that underlie resistance, we developed an approach to quantify the effects of all possible single nucleotide substitutions introduced into important regions of NA. We determined the experimental fitness effects of 450 nucleotide mutations encoding positions both surrounding the active site and at more distant sites in an N1 strain of IAV in the presence and absence of oseltamivir. NA mutations previously known to confer oseltamivir resistance in $\mathrm{N} 1$ strains, including $\mathrm{H} 275 \mathrm{Y}$ and N295S, were adaptive in the presence of drug, indicating that our experimental system captured salient features of real-world selection pressures acting on NA. We identified mutations, including several at position 223 , that reduce the apparent affinity for oseltamivir in vitro. Position 223 of NA is located adjacent to a hydrophobic portion of oseltamivir that is chemically distinct from the substrate, making it a hotspot for substitutions that preferentially impact drug binding relative to substrate processing. Furthermore, two NA mutations, K221N and Y276F, each reduce susceptibility to oseltamivir by increasing NA activity without altering drug binding. These results indicate that competitive expansion of IAV in the face of drug pressure is mediated by a balance between inhibitor binding and substrate processing.
\end{abstract}

Keywords: experimental fitness, systematic mutation, adaptive, neuraminidase inhibitor, oseltamivir 


\section{Introduction}

Influenza A virus (IAV) causes a highly contagious acute respiratory illness responsible for significant morbidity and mortality in humans. IAV has two surface glycoproteins, hemagglutinin (HA) and neuraminidase (NA) that are used to distinguish subtypes. The most common IAV subtypes that infect humans are H1N1 and H3N2. H1N1 IAV has caused several influenza pandemics, including the 1918 Spanish flu and the 2009 swine flu [1, 2]. HA binds to sialic acid that is part of glycoproteins located on the surface of host cells and is critical for initial attachment and infection. NA cleaves sialic acid from host cell glycoproteins during the release of newly formed viral progeny, thus reducing viral affinity for previously infected cells [3]. The NA competitive inhibitor oseltamivir is widely used for treatment of influenza [4]. Oseltamivir is a successful example of structure-based drug design, in which electrostatic interactions have been optimized between the drug and the protein [5]. As NA is an enzyme, the active site is more conserved than the rest of the protein surface to preserve the necessary activity for viral release [6]. Yet antiviral resistance is a persistent problem with IAV; the use of oseltamivir to prevent morbidity and mortality has been disappointing due to widespread drug resistance [79]. Improved approaches to combat influenza infection and an increased understanding of drug resistance mechanisms are in great demand.

Clinical reports have shown the emergence of a handful of different oseltamivirresistance mutations in H1N1 IAV following the clinical use of oseltamivir [9]. Thus far, mutations that have been associated with oseltamivir resistance occur at only a few positions that neighbor the NA active site [10-13]. The most prevalent resistance mutation in H1N1 IAV encodes the $\mathrm{H} 275 \mathrm{Y}$ substitution (N1 numbering system used throughout), which spread globally 
in 2008 [8]. Most oseltamivir resistance mutations that have been studied cause defects in viral expansion in the absence of drug pressure $[14,15]$. For example, the H275Y mutation that is commonly observed in H1N1 isolates with oseltamivir resistance, caused a reduced titer in the absence of drug in the WSN strain [16]. In the case of H275Y, secondary mutations including R222Q and V234M can restore fitness and the combined H275Y/R222O/V234M genotype became predominant in circulating H1N1 in 2008 [17]. The analyses of individual IAV clones indicate that costs of adaptation can mediate the mutations that emerge in response to drug pressure. Because only a limited number of mutations have been studied, often in different strain backgrounds, the extent to which fitness costs mediate the emergence of drug resistance mutations in IAV is unclear.

To effectively probe drug resistance mechanisms, precise measurements of the effects of individual mutations are critical because small differences can distinguish mutants that will have different evolutionary outcomes. This remains a technical challenge despite exciting recent findings from gene-wide analyses of mutant effects in IAV [18-23] and genome-wide analyses in poliovirus [24]. Gene-wide studies of mutations in IAV provide useful estimates of the average impact of mutations at each amino acid position and effectively delineate the strength of selection acting at each position in the NA, NS, NP and HA genes of IAV. However, the effects of specific amino acid substitutions from gene-wide analyses of IAV $[18,20,21]$ are only modestly reproducible $\left(\mathrm{R}^{2}\right.$ ranging from 0.34 to 0.62$)$.

To measure fitness effects in IAV with high precision, we adapted the EMPIRIC (Exceedingly Meticulous and Parallel Investigation of Randomized Individual Codons) approach that we previously developed to investigate fitness landscapes in yeast [25-31]. The EMPIRIC 
approach, along with related approaches developed by others [32-35], utilizes bulk competitions of engineered mutational libraries and next generation sequencing to estimate the frequency of each mutation before and after selection. A similar strategy used by Sun and colleagues was successful in identifying resistance mutations in the NS5A gene of hepatitis C virus [36]. Here, we adapted the EMPIRIC approach to systematically quantify the fitness effects of mutations in the NA gene of influenza A/WSN/33, which is an $\mathrm{H} 1 \mathrm{~N} 1$ strain. The results of this study provide improved precision relative to other high throughput studies of IAV mutants and enabled a robust assessment of drug resistance and fitness costs in the absence of drug pressure.

Our results indicate that a balance between mutant effects on binding to drug and processing of substrates mediates drug resistance mutations. In the absence of drug pressure, most mutations exhibited fitness defects. The presence of oseltamivir changed the fitness effects of many mutations including a handful that became adaptive. The strongest drug adaptive mutations (H275Y, N295S, and I223M) have previously been associated with drug resistance in clinical $\mathrm{N} 1$ isolates $[8,37,38]$. These drug adaptive mutations had similar fitness defects (30-33\%) compared to the parental strain in the absence of oseltamivir. In contrast, mutations associated with drug resistance in clinical N2 isolates caused severe fitness defects when introduced into the N1 strain used in our experiments $(60-100 \%$ defects relative to the parental N1 strain in the absence of drug) that hinder them from being adaptive in N1. These observations suggest that fitness costs govern the resistance mutations that emerge in different IAV subtypes. We observed the vast majority of mutants at position 223 became adaptive to oseltamivir and had decreased drug binding. We also identified two drug adaptive mutations, 
K221N and Y276F that did not decrease drug binding, but increased the efficiency of substrate processing. These observations demonstrate that resistance to oseltamivir can occur by two distinct mechanisms: decreased binding to drug or increased efficiency of substrate processing.

\section{Results}

We analyzed the fitness effects of all single nucleotide mutations in five specific 30 base regions of NA, focusing on mutations encoding regions of NA immediately adjacent to the active site as well as a control region on the surface of NA far from the active site (Table 1 and Fig. 1A). Regions around the active site were selected to include amino acid positions previously associated with oseltamivir resistance in human isolates, as well as positions that could encompass potentially novel mutations. Mutations were site-specifically engineered into plasmid-encoded viral genomes using a previously described plasmid system in which each influenza gene segment of the A/WSN/33 H1N1 strain is encoded on an individual plasmid [39]. Libraries of NA plasmids containing all possible single nucleotide mutations at 30 consecutive bases were generated. The NA plasmid libraries were combined with plasmids containing the remaining seven segments and transfected into co-cultured 293T and Madin-Darby canine kidney (MDCK) cells to recover an initial viral pool containing engineered mutations (P0) (Fig. 1B). This pool was used to infect MDCK cells in the absence or presence of oseltamivir, a competitive inhibitor of NA. P1 viruses were isolated from these infected MDCK cells.

\section{Systematic approach to quantify the fitness effect of NA mutants with high precision}


We analyzed a total of 50 amino acid positions in NA. The frequency of each mutation in the plasmid, P0, and P1 samples provided a direct estimate of the fitness effects of all 450 single nucleotide mutations in these regions without drug pressure (Table S1 and S2). Stop codons were universally depleted in P0 samples relative to plasmid. In contrast, synonymous mutations were relatively unchanged in frequency between plasmid, P0, and P1 pools (Fig. S1). For mutations whose frequencies could be assessed in the plasmid, P0, and P1 pools, we observed consistent changes in frequency during viral recovery (plasmid to P0) and viral propagation (P0 to P1) (Fig. S2). These observations indicate that similar selection occurred during P0 viral recovery and P1 viral propagation. To compare the effects of mutations in different experiments with potential region-specific bias, we normalized fitness effects, setting the average stop codon for each bulk competition to -1 to represent null NA function and average synonymous substitutions in each competition to 0 to represent WT-like fitness. We estimated fitness effects of mutations independently from changes in frequency from plasmid to P0, and from P0 to P1.

Fitness estimates for mutations based on P1 analyses in the absence and presence of drug are shown in Fig. $1 \mathrm{C}$ and Fig. 1D, respectively, and results were highly reproducible with $\mathrm{R}^{2}$ $>0.9$ for experimental replicates of viral expansion. Comparing bulk analyses with mutations analyzed in isolation is an important control that probes both stochastic noise and potential systematic differences between these independent approaches. We performed fitness analyses on a panel of 22 clones of individual mutations and observed strong correlation with fitness estimates from our bulk analyses (Fig. 1E and Fig. S3). These observations indicate that our bulk studies are accurate estimates of fitness effects of isolated clones. 


\section{Fitness effects of mutations in NA without drug pressure}

We examined how missense mutations that change the amino acid sequence impact fitness in the absence of drug pressure (Fig. 2A). The vast majority of amino acid changes in regions of NA close to the active site caused severe fitness defects. In contrast, many amino acid changes in the control region located far from the active site were compatible with robust fitness (Fig. 2B). These observations are consistent with the intuition that enzyme catalysis is sensitive to the physical composition of the amino acids surrounding the active site. To estimate the sensitivity of each amino acid position to mutation, we calculated the average fitness effect observed for missense mutations (Fig. S4A). This metric of mutational sensitivity correlated $\left(R^{2}=0.46\right)$ with solvent exposure (Fig. S4B), indicating that solvent exposure contributes to the sensitivity of a position to mutation.

We examined the relevance of these experimental fitness analyses of the WSN strain under laboratory conditions to natural evolution. The WSN strain was chosen for these experiments because it can be efficiently recovered from plasmids, which helped facilitate the recovery of diverse viral libraries necessary for the competition experiments. WSN was cloned from a strain originally isolated in 1933 that had subsequently been passaged under laboratory conditions. The NA gene of WSN contains an unusual deletion in the stalk region that has been observed to partially impair viral expansion in chicken eggs, but not in mammalian cells [40]. The sequence of amino acids that comprise the active site of NA are similar to circulating viruses. To investigate how lab adaptation of WSN may influence the interpretation of our results, we compared the experimental fitness effects of amino acid changes that we observed 
in WSN with the frequency of the same amino acids in 6,205 sequenced H1N1 isolates available from the influenza research database [41] (Fig. 2C).

The amino acids most frequently sampled in the sequenced $\mathrm{H} 1 \mathrm{~N} 1$ isolates, including the most frequent amino acid at each position that we experimentally analyzed, all supported robust viral expansion when generated in the WSN genetic background (Fig. 2C). In contrast, the vast majority of mutations that caused severe experimental fitness defects in WSN were either never or rarely observed in sequenced H1N1 isolates. Multiple factors could contribute to the observation that some mutations with severe fitness defects in WSN were rarely observed in sequenced isolates including a strong dependence on genetic background for these mutations as well as potential rare errors in the sequencing process of these isolates. Overall, the correspondence we observe between WSN fitness effects and the frequency of amino acids observed in sequenced H1N1 isolates suggests that our analyses of NA mutations in WSN capture many salient features of natural selection acting on the NA protein of circulating H1N1 IAV.

To investigate the extent to which our fitness experiments in WSN could extend to other IAV strains, we compared them to the frequency of amino acids observed in 5,279 sequenced H3N2 isolates available from the influenza research database [41] (Fig. 2D). Many of the most common amino acids identified in H3N2 sequenced isolates were moderately to strongly deleterious when generated in WSN. This observation indicates that strongly epistatic or context-dependent amino acid substitutions have accrued during the divergence of the N1 and N2 proteins. 


\section{N1 and N2 subtype specific mutant effects}

To further examine how divergence of N1 and N2 proteins contribute to fitness effects of mutants in different genetic backgrounds, we analyzed the mutations E119V and R293K, which have been specifically associated with oseltamivir resistance in N2 isolates. Both E119V and R293K had strong fitness defects when generated in the N1 of WSN: E119V was lethal and R293K exhibited a $62 \%$ defect relative to the parental WSN strain without drug pressure. We attempted to recover viral stocks from individually cloned plasmids encoding E119V and R293K in WSN, but neither yielded measureable titers (data not shown). The failure to recover viral stocks of these variants is consistent with the severe fitness defects observed for these mutations in our bulk competitions. A previous study demonstrates that oseltamivir binding is decreased by the E119V mutation in the WSN strain and showed that E119V dramatically reduces the susceptibility of WSN NA to oseltamivir inhibition compared to both the parental wild type NA as well as the $\mathrm{H} 275 \mathrm{Y}$ variant that is most commonly associated with oseltamivir resistance in the N1 strain[42]. In the same study, the enzymatic activity of the R293K mutation was too low to be accurately measured by the NA inhibition experiment [42]. When expressed in 293T cells, both the E119V and R293K variants of WSN NA had enzyme activity in the absence of oseltamivir that was less than $5 \%$ that observed for wild type NA in the absence of oseltamivir (Fig. 2E). These observations suggest that severe fitness costs in the absence of oseltamivir prevent E119V and R293K from contributing to drug adaptation in WSN. In future research beyond the scope of this work, it will be of interest to investigate the process by which N1 and N2 proteins have accumulated context-dependent amino acid substitutions. 


\section{Fitness effects of mutations in NA with oseltamivir}

To examine the impact of drug pressure on NA mutations, we compared fitness effects observed with and without oseltamivir (Fig. 3A). To provide a sensitive readout, we utilized a concentration of drug $(1 \mu \mathrm{M})$ that partially reduced expansion of the parental WSN strain. Using a Student's $t$-test with multiple test correction, we identified 24 drug responsive mutations whose fitness effect was significantly increased in the presence of this concentration of oseltamivir (Table S3; plotted in orange in Fig. 3A). Most of these drug responsive mutations exhibited fitness defects without drug, indicating that they reduce substrate processing as well as drug binding. The fitness defects of many of the drug responsive mutations hindered their ability to outcompete the parental strain under the conditions of these experiments. Using a similar statistical approach, we determined that only five mutations (K221N, I223M, H275Y, Y276F, and N295S, N1 numbering system) were significantly more fit than the parental strain in the presence of $1 \mu \mathrm{M}$ oseltamivir, and we refer to these mutations as drug adaptive.

Three of these five drug adaptive mutations from our experiments (H275Y, N295S, and I223M) have previously been associated with oseltamivir resistance (Table 2 ) [9, 38, 43]. In our experiments, $\mathrm{H} 275 \mathrm{Y}$ was the strongest drug resistance mutation, with greater than two-fold increased fitness in the presence of oseltamivir compared to wild-type (Fig. $3 \mathrm{~A}$ ). $\mathrm{H} 275 \mathrm{Y}$ is the predominant oseltamivir resistance mutant that has appeared in seasonal and pandemic H1N1 influenza virus [9]. The N295S mutation, which exhibited a 50\% fitness improvement in the presence of oseltamivir compared to wild-type in our experiments, has also been associated with oseltamivir resistance in different serotypes of IAV, although less frequently than $\mathrm{H} 275 \mathrm{Y}$ $[37,43,44]$. The I223M mutation has been associated with oseltamivir resistance in H5N1 IAV 
[38]. These three drug adaptive mutations (H275Y, N295S, and I223M) all had similar fitness defects ranging from $30-33 \%$ relative to the parental strain in the absence of drug. These observations indicate that the influenza virus can tolerate large fitness costs in the face of a novel selection pressure. In principle, fitness defects of this magnitude impose subsequent selection for compensatory mutations that can restore substrate processing and fitness. This scenario has been observed with influenza viruses carrying the $\mathrm{H} 275 \mathrm{Y}$ mutation that accumulated the compensatory R2220/V234M mutations and became the dominant circulating strain in 2008 [17].

To examine if mutations with more extreme fitness costs could outcompete the parental strain at different drug concentrations, we measured the fitness effects of mutations at positions 292-301 under a range of oseltamivir concentrations (Table S4). This region includes two drug responsive mutations with different fitness costs relative to the WSN virus without oseltamivir: N295S (30\% fitness cost) and R293K (70\% fitness cost). These mutations both exhibited fitness effects that increased with oseltamivir concentration (Fig. 3B), supporting the conclusion that these mutations reduce susceptibility to oseltamivir. While we observed fitness effects responding to oseltamivir concentration, the changes in fitness effects were small (2fold) relative to the changes in oseltamivir concentration (16-fold). Over this range of oseltamivir concentrations, the R293K mutation is always less fit than the parental strain. These observations suggest that the strong fitness cost of the R293K mutation hinders its ability to outgrow the parental strain and cause drug resistance.

We investigated how the fitness effects of mutations based on bulk competitions in the presence and absence of oseltamivir compared with analyses of individual clones of five 
variants (Fig. 3C). This comparison is complicated because different references are utilized in each experiment: in bulk competitions, the fitness of mutations are determined directly relative to $\mathrm{WT}$, and in the analyses of individual clones the effect of drug is relative to the expansion rate of each viral variant in the absence of drug. The response of an isolated clone to oseltamivir when not in competition with other viruses should be a function of fitness effects measured in bulk competitions both with oseltamivir and in the absence of drug. Consistent with this principle, the order of the sensitivity of four isolated clones to oseltamivir (Fig. 3C) was the same as the order of mutations based on the difference between fitness effects with and without oseltamivir (Y276F < 1223M < N295S < H275Y).

\section{Position 223 is a hotspot for mutations that decrease binding to oseltamivir}

Hotspots have been associated with drug resistance in many systems [45, 46]. To scan for potential hotspots that impact oseltamivir binding relative to substrate processing, we calculated the average drug responsiveness (defined as change in average fitness effect of mutations with oseltamivir compared to without drug) at each analyzed position (Fig. 4A). Mutations at position 223 and 275 exhibited the strongest averaged oseltamivir responsiveness. Analyzing the individual mutations at these positions indicated that the responsiveness of position 275 was almost completely due to one mutation (H275Y) of very large effect (Fig. 4B). In contrast, the responsiveness of position 223 was due to multiple mutations with intermediate drug responsive effects.

To investigate the drug responsive effects of mutations at position 223 in further detail, we generated individual clones and examined their responsiveness to oseltamivir in vitro (Fig. 
4C). We observed that six different mutations at position 223 (Arg, Lys, Met, Leu, Thr, and Val) were less sensitive to oseltamivir than WT. All of the mutations at position 223 that we analyzed were more sensitive to oseltamivir than the $\mathrm{H} 275 \mathrm{Y}$ mutation. Together, our results indicate that a very specific physical change at position 275 is required to disrupt drug binding. This is consistent with structural analyses of $\mathrm{H} 275 \mathrm{Y}$, which indicate that subtle conformational rearrangements mediated by the side chain of E277 that is located between position 275 and oseltamivir are responsible for disrupting drug binding [13]. While position 275 does not directly contact oseltamivir or substrate, position 223 is located at the substrate binding site and can directly contact oseltamivir (Fig. 4D). Position 223 contacts hydrophobic atoms in oseltamivir that are outside the substrate envelope and that differ in physical properties from the polar substrate. Distinctions between substrate and inhibitors have been associated with hotspots for drug resistance in HIV protease [47]. The differences between oseltamivir and substrate where these contact position 223 likely cause this position to be a hotspot for mutations that disrupt drug binding in NA. Of note, the I223K, I223R, and I223T mutations have been observed in $2009 \mathrm{H} 1 \mathrm{~N} 1$ isolates with reduced sensitivity to oseltamivir [48, 49], although other mutations at position 223 have not yet been associated with adaptation to oseltamivir in the H1N1 subtype to the best of our knowledge. Structural analyses of neuraminidase with I223R demonstrate that this mutation physically disrupts binding to oseltamivir [50].

\section{Drug adaptive mechanism of Y276F and K221N}

The Y276F and K221N mutations had similar or slightly improved fitness compared to the parental strain in the absence of drug pressure (Fig. 5A). In bulk competition experiments, 
K221N exhibited smaller differences in fitness effects with and without drug compared to Y276F. The small fitness differences observed for K221N in bulk competitions would be difficult to discern with experiments on an individual clone. For these reasons, conclusions regarding K221N must be considered cautiously. Y276F exhibited a larger fitness increase and in isolation we observed that it did require elevated concentrations of oseltamivir to slow expansion relative to WT (Fig. 3C). Taken together, our observations indicate that Y276F improves the fitness of WSN both in the presence and absence of oseltamivir. To the best of our knowledge, the Y276F mutation has not previously been associated with oseltamivir resistance.

To investigate potential drug adaptive mechanisms of K221N and Y276F, we closely examined structural and biochemical properties of each mutation. The $C \alpha-C \beta$ bond of amino acids at positions 221 and 276 are oriented away from the active site (Fig. 5B) such that the side chains are unlikely to directly contact either the inhibitor oseltamivir or the sialic acid moiety of the substrate. This observation suggests that the fitness effects of the $\mathrm{K} 221 \mathrm{~N}$ and $\mathrm{Y} 276 \mathrm{~F}$ mutations are likely due to subtle alterations to the conformation or dynamics of nearby positions that do contact substrate and inhibitor.

We analyzed the enzymatic activity of K221N and Y276F as well as the other three identified drug adaptive mutations (I223M, H275Y, and N295S) in vitro using a fluorescent substrate (Fig. 5C). The three drug-adaptive mutations with fitness costs in the absence of drug (I223M, H275Y, and N295S) all had reduced enzymatic activity relative to WT. These results suggest that reduced substrate turnover caused by these mutations is responsible for decreased fitness in the absence of drug pressure. In contrast, K221N and Y276F both exhibited increased enzymatic activity relative to WT. In principle, mutations that increase enzymatic 
activity without impacting drug binding can provide an adaptive advantage in the face of drug pressure. For example, a mutation that increases the efficiency of substrate processing two-fold without impacting drug binding will increase WT enzymatic activity in the presence of drug concentrations that reduce the WT enzyme efficiency 2 -fold. To examine if this mechanism is relevant to the $\mathrm{K} 221 \mathrm{~N}$ and $\mathrm{Y} 276 \mathrm{~F}$ mutations, we analyzed the effects of these mutations on inhibition by oseltamivir in vitro (Fig. 5D). Full experimental titration experiments with WT indicate that the results of this inhibition assay are highly reproducible. Both $\mathrm{K} 221 \mathrm{~N}$ and Y276F had inhibition profiles and 50\% enzyme-inhibitory concentration $\left(\mathrm{IC}_{50}\right)$ values that were similar or slightly more sensitive to oseltamivir than WT (Fig. 5D). These results indicate that the adaptive advantage of $\mathrm{K} 221 \mathrm{~N}$ and $\mathrm{Y} 276 \mathrm{~F}$ are due to increased efficiency of substrate processing rather than decreased binding to drug.

\section{Discussion}

Many pathogens, including IAV, accumulate mutations that make them resistant to currently available drugs. While some mutations that cause influenza to become resistant to oseltamivir have been identified, the impact of most mutations has not been fully resolved. In particular, the effect of most mutations in the absence of drug pressure has not been experimentally characterized. Here, we systematically analyzed all possible single nucleotide mutations in regions of the active site of the viral NA gene using the EMPIRIC method. Our approach provides rapid and highly reproducible fitness measurements of IAV mutants. We comprehensively examined fitness effects of all possible single nucleotide mutations in defined 
regions of NA in the absence and presence of oseltamivir, identifying mutations that confer resistance to oseltamivir and validating mutations with individual clones.

Our results support the conclusion that a balance between substrate processing and drug binding determines the potential of an NA mutation to adapt to oseltamivir (Fig. 6A). Mutations with either decreased drug binding (Fig. 6B, purple line) or increased substrate processing (Fig. 6B, green line) should expand more efficiently compared to WT in the presence of drug (Fig 6B, black line). In principle, mutations that decrease drug binding without causing a defect in substrate processing provide the ability for viruses to expand most efficiently over the broadest concentrations of drug (Fig. 6B). Multiple lines of evidence indicate that this type of mutation is extremely rare: none were identified in our mutational scan, and drug resistance mutations identified in clinical isolates exhibit fitness costs without drug [14, 15]. Instead, most mutations associated with oseltamivir resistance in clinical isolates had clear experimental fitness defects ( $\sim 30 \%)$ in our experiments (Fig. 6B, red line). The fitness defects of these drugresistant mutations should impose selection for compensatory mutations that increase the efficiency of substrate processing, which has been observed both experimentally [51] and in the majority of H1N1 clinical isolates from 2008 [17].

Our results indicate that NA mutations can also provide an adaptive advantage in the presence of oseltamivir by increasing the efficiency of substrate processing without decreasing sensitivity to drug. The Y276F mutation that exhibited the largest increase in substrate processing efficiency and fitness effect with oseltamivir has been observed at low frequency (0.1\%, only observed in 2009-2010) in sequenced H1N1 isolates. The low frequency of Y276F in sequenced $\mathrm{H} 1 \mathrm{~N} 1$ isolates suggests that the effects of $\mathrm{Y} 276 \mathrm{~F}$ may be distinct in different $\mathrm{N} 1$ 
sequence backgrounds. Nonetheless, our observations of the effects of Y276F in WSN highlight the general mechanistic conclusion that increased efficiency of substrate processing by NA without reduced sensitivity to oseltamivir binding can lead to drug adaptation. While this type of mutation does not provide resistance to high levels of oseltamivir, it may act as an enabling mutation that provides greater sampling of secondary mutations that could lead to strong drug resistance. For example, a mutation that increases the efficiency of substrate processing may offset the fitness costs of a mutation that reduces binding to drug and processing of substrate such that the double mutant disrupts binding to drug while maintaining efficient substrate processing.

Our results, as well as other studies $[17,52]$, indicate that multiple mutations in NA are required to reduce oseltamivir binding while maintaining efficient substrate processing. The evolution of drug resistance in this scenario will be a complex combination of mutational probabilities as well as the effects of individual and combined mutations on binding to drug and processing of substrate. Additional studies of combinations of mutations will be critical to appreciate the full spectrum of potential oseltamivir resistance NA variants. This should also serve as a caution that widespread use of oseltamivir at sub-neutralizing concentrations will likely lead to an increased frequency of multiple NA mutations with adaptive benefits.

\section{Materials and Methods}

\section{Construction of plasmid-encoded libraries}

NA point mutant libraries were generated using a previously described approach [26, 53]. Plasmids encoding the parental NA gene as well as the other seven gene segments 
encoding the H1N1 A/WSN/33 strain in the pHW2000 vector were kindly provided by R.

Webster (St. Jude Children's Research Hospital, Memphis, TN). The NA gene was cloned into pRNDM to generate a plasmid without any Bsal restriction sites. Inverted Bsal sites were then introduced to enable a cassette ligation strategy to efficiently generate libraries of single nucleotide mutations; a separate cassette was used to mutate each base to all three nonparental bases. Libraries of single nucleotide mutants at 30 consecutive bases were combined and blended with parental (wild-type) plasmid as well as a panel of three stop codons at about 4-fold elevated frequency as negative controls for NA function. The resulting pool of NA libraries was transferred from pRNDM to pHW2000 [39] using sequence and ligation independent cloning (SLIC) $[53,54]$. The pHW2000 construct contains a CMV promoter to drive expression of mRNA and a polll promoter in the opposite orientation to generate genomic negative strand RNA.

\section{Cell culture}

$293 T$ and MDCK cell lines were obtained from the American Type Culture Collection (Manassas, VA). The 293T cell line was maintained in 293T cell culture media consisting of OptiMEM I reduced serum media (Gibco, Grand Island, NY) supplemented with 5\% fetal bovine serum (Hyclone, Logan, UT), $100 \mathrm{U} / \mathrm{mL}$ penicillin, and $100 \mu \mathrm{g} / \mathrm{mL}$ of streptomycin at $37^{\circ} \mathrm{C}$ and 5\% carbon dioxide. The MDCK cell line was maintained in Eagle's minimal essential medium (MEM) supplemented with $10 \%$ fetal bovine serum, $2 \mathrm{mM}$ L-glutamine, $10 \mathrm{mM}$ sodium pyruvate, $1 \mathrm{X}$ non-essential amino acid, $100 \mathrm{U} / \mathrm{mL}$ penicillin, and $100 \mu \mathrm{g} / \mathrm{mL}$ of streptomycin at 


\begin{abstract}
$37^{\circ} \mathrm{C}$ and $5 \%$ carbon dioxide. All cell culture reagents were from Corning (Manassas, VA) unless otherwise indicated.
\end{abstract}

\title{
Viral library recovery and selection experiments
}

Viral libraries were recovered from plasmids as previously described [39]. Briefly, equal numbers of 293T and MDCK cells were mixed in 293T cell culture media, and seeded in 6-well plates at a density of $2-6 \times 10^{5}$ cells/well. 293T-MDCK co-cultures were transfected with $1 \mu \mathrm{g}$ of NA plasmid library and $1 \mu \mathrm{g}$ of each plasmid encoding the other seven gene segments (8 $\mathrm{gg}$ total plasmid) using TransIT-LT1 Reagent (Mirus, Madison, WI). The ratio of DNA ( $\mu$ g) to TransITLT1 $(\mu \mathrm{L})$ was 1:2. At 6 hours post-transfection, cell growth media was replaced with fresh OptiMEM I reduced serum media. At 30 hours post-transfection, TPCK-trypsin (Sigma-Aldrich, St. Louis, MO) was added to cell growth media to a final concentration of $0.5 \mu \mathrm{g} / \mathrm{mL}$. At 72 hours post-transfection, supernatant containing viral particles was harvested and centrifuged at $300 x g$ for 15 minutes to remove cell debris. Supernatants were stored at $-80^{\circ} \mathrm{C}$. These recovered pools of viral variants are referred to as PO libraries. Plaque assays were performed to determine the titer (plaque forming units or PFU/mL) of each P0 sample as previously described [55].

MDCK cells were used for additional viral competition experiments. $10^{6} \mathrm{MDCK}$ cells were seeded in individual wells of a 6-well plate and grown for one day. Cells were washed twice with 1X PBS and once with cDMEM/BSA (DMEM, $100 \mathrm{U} / \mathrm{mL}$ penicillin, $100 \mu \mathrm{g} / \mathrm{mL}$ streptomycin, and $7.5 \% \mathrm{BSA}$ ) before infection. P0 viral libraries were diluted in influenza virus growth medium (IVGM: cDMEM/BSA with $1 \mu \mathrm{g} / \mathrm{mL}$ TPCK trypsin). MDCK cells were infected at a multiplicity of 
infection (MOI) of 0.001. For each experimental dataset, three independent infections were conducted in the presence or absence of $1 \mu \mathrm{M}$ oseltamivir. Replicate datasets were obtained by performing three additional independent P1 infections. Oseltamivir carboxylate was a kind gift from Hoffmann-La Roche Pharmaceuticals (Basel, Switzerland). Viral binding was conducted at $37^{\circ} \mathrm{C}$ with $10 \%$ carbon dioxide for one hour, followed by two washes with PBS. After washing, 2 $\mathrm{mL}$ of fresh IVGM was added to each well of MDCK cells, which were maintained at $37^{\circ} \mathrm{C}$ with $10 \%$ carbon dioxide. The supernatant containing viruses was collected when 50\%-90\% CPE was observed or at 120 hours post-infection. Supernatants were centrifuged at $300 x g$ for 15 minutes and stored at $-80^{\circ} \mathrm{C}$. Samples recovered from MDCK expanded viral pools were referred to as P1.

\section{Analyses of individual mutants}

A panel of individual NA variants were cloned into plasmids and analyzed in isolation. Viral samples were recovered from 293T-MDCK cells as described for library samples. Titers of P0 samples were determined for each variant using plaque assays. For variants that produced P0 samples with titers $>10,000 \mathrm{PFU} / \mathrm{mL}$, we also analyzed plaque size. Plaque size was measured using a Nikon SMZ1500 microscope for 20 randomly selected and well separated plaques for each variant analyzed (Fig. S3).

For a subset of variants, infectivity was analyzed as a function of oseltamivir concentration as previously described [56]. Briefly, confluent MDCK cells in 24-well plates were infected at an $\mathrm{MOI}$ of 0.01 . The infection was conducted in a range of oseltamivir concentrations $(0,0.1,0.3,1,3,10,30$ and $100 \mu \mathrm{M})$, and incubated at $37^{\circ} \mathrm{C}$ with $10 \%$ carbon 
dioxide. Supernatants were collected 3 days post-infection and virus titer was determined by plaque assay.

\section{Analyses of enzyme activity in vitro}

Enzyme activity of NA was determined using fluorogenic 2'-(4-methylumbelliferyl)-alpha-D-Nacetylneuraminic acid (MUNANA) substrate according to the manufacturer's instructions (Life Technologies, Carlsbad, CA). Briefly, recombinant viruses were incubated with MUNANA substrate at a final concentration of $0.1 \mathrm{mM}$ for one hour at $37^{\circ} \mathrm{C}$ with shaking. After this incubation, fluorescence was measured using a Victor X5 plate-reader (PerkinElmer, Waltham, MA) with a $355+/-10 \mathrm{~nm}$ excitation filter and a $460+/-20 \mathrm{~nm}$ emission filter. RFU was normalized to the titer of viruses determined by plaque assay to obtain estimates of relative NA activity between different variants. To estimate sensitivity to oseltamivir, MUNANA assays were performed in the presence of a range of oseltamivir concentrations. Recombinant viruses were incubated with various concentrations of oseltamivir for 45 minutes at $37^{\circ} \mathrm{C}$ with shaking and then reacted with MUNANA as described. The fluorescent signal at each concentration of oseltamivir was normalized to the signal in the absence of oseltamivir and the resulting data fit to a standard binding equation in order to estimate $\mathrm{IC}_{50}$ values. Enzymatic activity of $\mathrm{E} 119 \mathrm{~V}$ and R293K was estimated by transiently expressing NA on the surface of $293 \mathrm{~T}$ cells according to a previously published protocol with modifications [17]. Briefly, an equal amount of plasmid harboring WT or mutant NA was transfected into an equal number of 293T cells using TransITLT1 Reagent (Mirus, Madison, WI). 293T cells were harvested 24 hours post transfection and resuspended in non-lysis buffer before subject to MUNANA assays as described for 
recombinant viruses. Sensitivity of I223R and I223K to oseltamivir was also estimated by expressing NA in 293T cells and then incubated with oseltamivir, followed by reaction with MUNANA as described. $I_{50}$ values were estimated by fitting normalized signal to a standard binding equation.

\section{Sequence analyses}

Influenza genomic RNA was extracted from supernatants containing virions using the QIAamp Viral RNA Mini Kit (Qiagen, Germantown, MD). Viral RNA was reverse transcribed into cDNA using primers binding upstream of randomized libraries and SuperScriptIII (Life Technologies, Beverly, MA). Subsequent processing steps were as described previously for analyzing mutant frequency [26]. Briefly, samples were barcoded to distinguish replicates as well as plasmid, $\mathrm{PO}$, and $\mathrm{P} 1$ samples and submitted for Illumina $36 \mathrm{bp}$ single read sequencing on a Genome Analyzer II. $2.05 \times 10^{7}$ high quality reads ( $>99.5 \%$ confidence across all 36 bases) were obtained and analyzed. Raw sequencing data has been submitted to the NIH Short Read Archive under accession number: Bioproject PRJNA272490 or SRA SRX839403. Read abundance $(R)$ is the count of each mutant. The relative abundance $(A)$ of each point mutant in plasmid or P0 or P1 library was estimated from logarithmic frequency of mutant normalized to WT, as indicated below in Equation 1.

$$
A=\log _{2}\left(\frac{R_{m u t}}{R_{W T}}\right) \quad \text { (Eq. 1) }
$$

The frequency change $(F)$ of a mutation from P0 to the mean of three P1 replicates (Equation 2) was used as an estimate of the enrichment or depletion during viral expansion. Estimates of 
selection during virus recovery were made by comparing frequency changes between plasmid and PO using the same equations.

$$
F=A_{\overline{P 1}}-A_{P 0}
$$

Selection coefficients $(s)$ representing the experimental effects of each mutation on viral replication were calculated by normalizing the average stop codon to -1 (representing null fitness) and wild-type synonyms to 0 (representing no fitness effect), as indicated in Equation 3. Mutants with $s$ less than 0 had a fitness defect, whereas mutants with $s$ greater than 0 had a fitness benefit, relative to the parental sequence.

$$
s=\frac{F_{m u t}-F_{\overline{W T s y n}}}{F_{\overline{W T s y n}}-F_{\overline{\text { stop }}}}
$$

The above analyses yielded experimentally reproducible estimates of fitness effects for mutations with frequency greater than $0.2 \%$ (Fig. S5). Mutations below this frequency in P0 were likely subject to highly stochastic sampling in the pool of viruses used to start P1 passages. The majority of mutations with low frequency in PO were prevalent at far greater abundance in the plasmid-encoded libraries. These observations indicated that selection during viral recovery depleted viruses with unfit NA mutations, and is consistent with expected viral expansion in MDCK cells during the co-culture viral recovery to generate P0 samples. We observed that the frequency change of mutations from plasmid to P0 correlated $\left(R^{2}=0.89\right)$ with frequency changes from P0 to P1 (Fig. S2). From these observations we infer that selection pressure on NA mutations during plasmid to P0 rescue mimics selection during P0 to P1 expansion and that for mutations at high relative abundance in $\mathrm{P} 0$, frequency changes from $\mathrm{P} 0$ to $\mathrm{P} 1$ may be more accurate estimates of fitness effects than frequency changes from plasmid to P1. For these reasons, we calculated fitness effects without drug pressure for mutations with low frequency 
$(<0.2 \% ; 14 \%$ of our dataset) based on frequency changes from plasmid to P0, while for mutations that were abundant in PO ( $>0.2 \%)$ fitness effects were calculated solely based on frequency changes from P0 to P1. The severe fitness defects for these mutations with low frequency in the absence of drug make it unlikely that they would contribute to drug resistance.

\section{Estimate of variation of experimental fitness measurements}

We assessed potential sampling limitations (e.g., bottlenecking) in our viral recovery and passaging experiments by analyzing stop codons and synonymous codons. Stop codons had consistent and strong depletion in P0 samples relative to plasmid, whereas synonymous mutations had very limited changes in frequency between these pools (Fig. S1). This indicates that selection on NA occurs during PO viral recovery (consistent with infection of co-cultured MDCK cells during this step) and that sampling of plasmid variants during viral recovery is sufficient to reproducibly sample the number of mutations in our libraries. If sampling were insufficient, stochastic depletion of some synonymous mutations due to random undersampling should have been observed. In addition, four mutants with strong depletion in P0 samples relative to plasmid were studied in isolation and had strongly reduced or even no infectivity in an independent experiment, suggesting that mutants became depleted in PO samples mainly because they had detrimental effects on viral infectivity.

The variation in fitness effects between EMPIRIC experimental repeats (Fig. S5) suggests that sampling during initial viral entry was the main source of variance between replicates. This sampling was sufficient to analyze all single nucleotide variants, but would hinder the investigation of more complex libraries (e.g., libraries of all possible amino acid substitutions). 
To calculate confidence intervals on estimates of fitness effects from mutants that passed the P0 cutoff, we used a pooled error function for each data set. This approach provides a more robust estimate of standard errors for each mutant than calculations of the standard error of the mean based on three measurements. Pooled error functions were obtained by a log-linear regression of the individual standard deviations as a function of mutant frequency in PO (Fig. S5). An evaluation of the resulting residuals supported the validity of the pooled error approach. Mutations whose standard error appeared as an outlier of the estimated error distribution, which indicates potentially different sources of error for these mutations, are identified in Table S2. To estimate noise from sample processing and sequencing, viral samples generated solely from wild-type NA were processed by identical procedures and sequenced as control (Table S1).

\section{Statistical analysis to determine oseltamivir responsive mutants}

$t$-tests using $2 \times(\mathrm{N}-1)$ degrees of freedom (where $\mathrm{N}$ represents independent fitness measurements for each amino acid substitution in both drug and no-drug conditions) were used to compare fitness effects of amino acid substitutions with and without oseltamivir together with a multiple-test correction using a $5 \%$ false discovery rate.

\section{Analysis of mutant frequency in natural isolates}

$6205 \mathrm{H} 1$ N1 NA protein sequences and 5279 H3N2 NA protein sequences isolated from humans were downloaded from the Influenza Research Database [41]. These sequences were from viruses collected from 1933 to 2013, although the majority of viruses (76\%) were from the 
2004-2013. The dataset was curated to exclude partial sequences and duplicate entries.

Multiple sequence alignment was conducted using Multiple Sequence Comparison by Log-

Expectation, MUSCLE [57]. Positional amino acid frequencies were tabulated and compared to experimental fitness measurements.

\section{Acknowledgments}

We acknowledge the contributions of all members of the ALiVE (Algorithms to Limit Viral Epidemics) working group. We thank Melanie Trombly and Nese Kurt-Yilmaz for assistance with the preparation of the manuscript. This work was supported by the Prophecy Program, Defense Advanced Research Projects Agency, Defense Sciences Office [DSO], contract HR0011-11-C0095. 


\title{
Figure Legends
}

Figure 1. A high throughput approach was optimized to precisely analyze the fitness effects of NA mutations in influenza A virus. (A) Molecular image based on the PDB ID: 3B7E structure [60] of NA showing a monomer with the regions near the active site as well as a control region far from the active site that were chosen for mutational analysis highlighted in magenta. A competitive inhibitor bound in the active site of NA is shown as yellow spheres. (B) Site-directed mutagenesis was used to introduce point mutations into the plasmid encoding the NA gene from the influenza A/WSN/33 mixed with plasmids encoding wild-type versions of the other seven gene segments from this strain. Plasmids were co-transfected into 293T/MDCK cells to generate an initial PO library of viral particles that were subsequently expanded through infection of MDCK cells to generate a P1 library. Focused deep sequencing was utilized to quantify the enrichment or depletion of each mutation during viral expansion. (C) Reproducibility of high throughput estimates of fitness effects from experimental replicates of viral expansion. Silent substitutions (wild-type synonyms) in this plot are green and nonsense mutations (stop codons) are red. (D) Reproducibility of estimates of fitness effects in replicates of viral expansion with $1 \mu \mathrm{M}$ oseltamivir. (E) Correlation between fitness effects estimated for a panel of individual mutations analyzed in isolation with estimates from bulk competitions.

\author{
Figure 2. Fitness effects of mutants in WSN correspond to the frequency of amino acids \\ observed in sequenced H1N1 isolates. The distribution of fitness effects observed for \\ mutations in four regions proximal to the active site $(A)$ and one region distant from the active \\ site (B). Null-like and WT-like fitness effects are colored red and green and were based on
}


observations of stop codons and WT synonyms. Fitness effects of amino acid changes were compared to the frequency of amino acids observed in 6205 sequenced H1N1 isolates (C) and 5279 sequenced H3N2 isolates (D) available from the Influenza Research Database [41]. (E) Relative MUNANA activity of NA variants of WSN expressed in 293T cells monitored using a fluorescent substrate. Cells lacking the NA gene were included as a blank control. Error bars indicate the standard deviation of 3 biological replicates.

Figure 3. Oseltamivir adaptive mutations were identified by comparing fitness effects of NA mutations in the presence or absence of oseltamivir. (A) Comparison of the fitness effects of NA mutations with and without oseltamivir. Mutations with a statistically significant (detail in Materials and Methods) increase in fitness effect in the presence of oseltamivir compared to without drug (above and to the left of the diagonal) are colored orange. Mutations that were significantly more fit than WT in the presence of drug (above the horizontal dashed line) are labeled. (B) Fitness effects of two mutations associated with oseltamivir resistance in H1N1 (N295S) or H3N2 (R293K) from bulk competitions performed over a range of oseltamivir concentrations. The mutations exhibited fitness effects that increased with escalating amounts of oseltamivir. (C) Expansion of individually isolated mutations as a function of oseltamivir concentration.

Figure 4. Multiple mutations at position 223 reduced the apparent binding affinity of NA to oseltamivir. (A) To identify hotspots for mutations with adaptive potential to oseltamivir, we examined the average effect of mutations at each position on responsiveness to oseltamivir in 
the bulk competitions. (B) Oseltamivir responsiveness of single nucleotide mutations at positions 223 and 275. (C) Enzyme activity of individually isolated viral variants as a function of oseltamivir concentration. The activity of I223K and I223R was estimated in 293T cells while the activity of other mutants and WT was estimated using virus. (D) Structural image of an NA monomer indicating the location of positions 223 and 275 relative to oseltamivir and a substrate analog. The image was generated from PDB ID: 3CL2 [13] and PDB ID: 1F8B [61].

Figure 5. K221N and Y276F are adaptive in the presence of oseltamivir because they increase NA activity without altering drug binding. (A) Fitness effects of mutations that were statistically more fit than WT during bulk competitions with oseltamivir. Zero represents WT fitness, positive values represent increased fitness relative to $W T$, and negative values represent decreased fitness relative to WT. Error bar indicates standard error of the mean with $N=3$. (B) Structural image of an NA monomer indicating the location of positions 221 and 276 [53] relative to oseltamivir (blue) and a synthetic substrate used in enzymatic assays (yellow). The image was generated from PDB ID: 3CL2[13] and PDB ID: 1F8B [61]. (C) Relative activity of isolates of individual viral variants determined using the fluorescent substrate MUNANA. Enzyme activities were normalized to viral titer to estimate the relative enzyme activity per infectious unit. Error bars indicate the standard deviation of 5 biological replicates. The standard deviations are as follows: WT: 0.0499, Blank: 0.0002, E119V: 0.0004, R292K: 0.0001 (D) Enzyme activity of individually isolated viral variants as a function of oseltamivir concentration. The experimentally determined IC $50^{\prime}$ 's are as follows: WT: $0.89 \mathrm{nM}, \mathrm{K} 221 \mathrm{~N}: 1.04$ $\mathrm{nM}$, and Y276F: $0.55 \mathrm{nM}$. 
Figure 6. Mechanisms of adaptation to drug pressure. (A) Drug resistance is a balance between the effects of mutations on drug binding and substrate processing. Mutations that increase substrate processing or reduce drug binding favor drug resistance. (B) Theoretical model of the efficiency of substrate processing as a function of inhibitor concentration for mutations with different impacts. (C) Structural representation based on 3CL2.PDB of a NA subunit illustrating the location of mutations that either increased substrate processing (colored pink), or decrease binding to oseltamivir (colored green). Oseltamivir is shown in yellow and the catalytic tyrosine at position 406 is shown in cyan.

\section{References}

[1] Garten RJ, Davis CT, Russell CA, Shu B, Lindstrom S, Balish A, et al. Antigenic and genetic characteristics of swine-origin 2009 A(H1N1) influenza viruses circulating in humans. Science. 2009;325:197-201.

[2] Taubenberger JK, Kash JC. Influenza virus evolution, host adaptation, and pandemic formation. Cell Host Microbe. 2010;7:440-51.

[3] Wagner R, Matrosovich M, Klenk HD. Functional balance between haemagglutinin and neuraminidase in influenza virus infections. Rev Med Virol. 2002;12:159-66.

[4] von Itzstein M, Wu WY, Kok GB, Pegg MS, Dyason JC, Jin B, et al. Rational design of potent sialidasebased inhibitors of influenza virus replication. Nature. 1993;363:418-23.

[5] Lew W, Chen X, Kim CU. Discovery and development of GS 4104 (oseltamivir): an orally active influenza neuraminidase inhibitor. Curr Med Chem. 2000;7:663-72. 
[6] Gubareva LV, Kaiser L, Hayden FG. Influenza virus neuraminidase inhibitors. Lancet. 2000;355:827-

35.

[7] Dharan NJ, Gubareva LV, Meyer JJ, Okomo-Adhiambo M, McClinton RC, Marshall SA, et al. Infections with oseltamivir-resistant influenza A(H1N1) virus in the United States. Jama. 2009;301:1034-41.

[8] Moscona A. Global transmission of oseltamivir-resistant influenza. N Engl J Med. 2009;360:953-6.

[9] Samson M, Pizzorno A, Abed Y, Boivin G. Influenza virus resistance to neuraminidase inhibitors. Antiviral research. 2013;98:174-85.

[10] Collins PJ, Haire LF, Lin YP, Liu J, Russell RJ, Walker PA, et al. Structural basis for oseltamivir resistance of influenza viruses. Vaccine. 2009;27:6317-23.

[11] Oakley AJ, Barrett S, Peat TS, Newman J, Streltsov VA, Waddington L, et al. Structural and functional basis of resistance to neuraminidase inhibitors of influenza B viruses. J Med Chem. 2010;53:6421-31.

[12] Li Q, Qi J, Zhang W, Vavricka CJ, Shi Y, Wei J, et al. The 2009 pandemic H1N1 neuraminidase N1 lacks the 150-cavity in its active site. Nat Struct Mol Biol. 2010;17:1266-8.

[13] Collins PJ, Haire LF, Lin YP, Liu J, Russell RJ, Walker PA, et al. Crystal structures of oseltamivirresistant influenza virus neuraminidase mutants. Nature. 2008;453:1258-61.

[14] Ives JA, Carr JA, Mendel DB, Tai CY, Lambkin R, Kelly L, et al. The H274Y mutation in the influenza A/H1N1 neuraminidase active site following oseltamivir phosphate treatment leave virus severely compromised both in vitro and in vivo. Antiviral research. 2002;55:307-17.

[15] Herlocher ML, Truscon R, Elias S, Yen HL, Roberts NA, Ohmit SE, et al. Influenza viruses resistant to the antiviral drug oseltamivir: transmission studies in ferrets. The Journal of infectious diseases. 2004;190:1627-30.

[16] Abed Y, Goyette N, Boivin G. A reverse genetics study of resistance to neuraminidase inhibitors in an influenza A/H1N1 virus. Antivir Ther. 2004;9:577-81. 
[17] Bloom JD, Gong LI, Baltimore D. Permissive secondary mutations enable the evolution of influenza oseltamivir resistance. Science. 2010;328:1272-5.

[18] Bloom JD. An experimentally determined evolutionary model dramatically improves phylogenetic fit. Mol Biol Evol. 2014;31:1956-78.

[19] Heaton NS, Sachs D, Chen CJ, Hai R, Palese P. Genome-wide mutagenesis of influenza virus reveals unique plasticity of the hemagglutinin and NS1 proteins. Proc Natl Acad Sci U S A. 2013;110:20248-53.

[20] Thyagarajan B, Bloom JD. The inherent mutational tolerance and antigenic evolvability of influenza hemagglutinin. Elife. 2014;3.

[21] Wu NC, Young AP, Al-Mawsawi LQ, Olson CA, Feng J, Qi H, et al. High-throughput profiling of influenza A virus hemagglutinin gene at single-nucleotide resolution. Sci Rep. 2014;4:4942.

[22] Wu NC, Young AP, Al-Mawsawi LQ, Olson CA, Feng J, Qi H, et al. High-throughput identification of loss-of-function mutations for anti-interferon activity in the influenza A virus NS segment. J Virol. 2014;88:10157-64.

[23] Wu NC, Young AP, Dandekar S, Wijersuriya H, Al-Mawsawi LQ, Wu TT, et al. Systematic identification of $\mathrm{H} 274 \mathrm{Y}$ compensatory mutations in influenza A virus neuraminidase by high-throughput screening. J Virol. 2013;87:1193-9.

[24] Acevedo A, Brodsky L, Andino R. Mutational and fitness landscapes of an RNA virus revealed through population sequencing. Nature. 2014;505:686-90.

[25] Hietpas RT, Jensen JD, Bolon DN. Experimental illumination of a fitness landscape. Proc Natl Acad Sci U S A. 2011;108:7896-901.

[26] Hietpas R, Roscoe B, Jiang L, Bolon DN. Fitness analyses of all possible point mutations for regions of genes in yeast. Nat Protoc. 2012;7:1382-96.

[27] Roscoe BP, Bolon DN. Systematic exploration of ubiquitin sequence, E1 activation efficiency, and experimental fitness in yeast. J Mol Biol. 2014;426:2854-70. 
[28] Roscoe BP, Thayer KM, Zeldovich KB, Fushman D, Bolon DN. Analyses of the effects of all ubiquitin point mutants on yeast growth rate. J Mol Biol. 2013;425:1363-77.

[29] Jiang L, Mishra P, Hietpas RT, Zeldovich KB, Bolon DN. Latent effects of Hsp90 mutants revealed at reduced expression levels. PLoS Genet. 2013;9:e1003600.

[30] Bank C, Hietpas RT, Wong A, Bolon DN, Jensen JD. A bayesian MCMC approach to assess the complete distribution of fitness effects of new mutations: uncovering the potential for adaptive walks in challenging environments. Genetics. 2014;196:841-52.

[31] Hietpas RT, Bank C, Jensen JD, Bolon DN. Shifting fitness landscapes in response to altered environments. Evolution. 2013;67:3512-22.

[32] Fowler DM, Araya CL, Fleishman SJ, Kellogg EH, Stephany JJ, Baker D, et al. High-resolution mapping of protein sequence-function relationships. Nat Methods. 2010;7:741-6.

[33] Fowler DM, Fields S. Deep mutational scanning: a new style of protein science. Nat Methods. 2014;11:801-7.

[34] McLaughlin RN, Jr., Poelwijk FJ, Raman A, Gosal WS, Ranganathan R. The spatial architecture of protein function and adaptation. Nature. 2012;491:138-42.

[35] Fleishman SJ, Whitehead TA, Ekiert DC, Dreyfus C, Corn JE, Strauch EM, et al. Computational design of proteins targeting the conserved stem region of influenza hemagglutinin. Science. 2011;332:816-21. [36] Qi H, Olson CA, Wu NC, Ke R, Loverdo C, Chu V, et al. A quantitative high-resolution genetic profile rapidly identifies sequence determinants of hepatitis C viral fitness and drug sensitivity. PLoS Pathog. 2014;10:e1004064.

[37] Kiso M, Mitamura K, Sakai-Tagawa Y, Shiraishi K, Kawakami C, Kimura K, et al. Resistant influenza A viruses in children treated with oseltamivir: descriptive study. Lancet. 2004;364:759-65. 
[38] McKimm-Breschkin JL, Barrett S, Pudjiatmoko, Azhar M, Wong FY, Selleck P, et al. 1222

Neuraminidase mutations further reduce oseltamivir susceptibility of Indonesian Clade 2.1 highly pathogenic Avian Influenza A(H5N1) viruses. PLoS One. 2013;8:e66105.

[39] Hoffmann E, Neumann G, Kawaoka Y, Hobom G, Webster RG. A DNA transfection system for generation of influenza A virus from eight plasmids. Proc Natl Acad Sci U S A. 2000;97:6108-13.

[40] Castrucci MR, Kawaoka Y. Biologic importance of neuraminidase stalk length in influenza A virus. J Virol. 1993;67:759-64.

[41] Squires RB, Noronha J, Hunt V, Garcia-Sastre A, Macken C, Baumgarth N, et al. Influenza research database: an integrated bioinformatics resource for influenza research and surveillance. Influenza and other respiratory viruses. 2012;6:404-16.

[42] Abed Y, Baz M, Boivin G. Impact of neuraminidase mutations conferring influenza resistance to neuraminidase inhibitors in the N1 and N2 genetic backgrounds. Antivir Ther. 2006;11:971-6.

[43] Morlighem JE, Aoki S, Kishima M, Hanami M, Ogawa C, Jalloh A, et al. Mutation analysis of 2009 pandemic influenza $\mathrm{A}(\mathrm{H} 1 \mathrm{~N} 1)$ viruses collected in Japan during the peak phase of the pandemic. PLoS One. 2011;6:e18956.

[44] Le QM, Kiso M, Someya K, Sakai YT, Nguyen TH, Nguyen KH, et al. Avian flu: isolation of drugresistant H5N1 virus. Nature. 2005;437:1108.

[45] Zabriskie MS, Eide CA, Tantravahi SK, Vellore NA, Estrada J, Nicolini FE, et al. BCR-ABL1 compound mutations combining key kinase domain positions confer clinical resistance to ponatinib in $\mathrm{Ph}$ chromosome-positive leukemia. Cancer cell. 2014;26:428-42.

[46] Soumana DI, Ali A, Schiffer CA. Structural analysis of asunaprevir resistance in HCV NS3/4A protease. ACS chemical biology. 2014;9:2485-90.

[47] Ali A, Bandaranayake RM, Cai Y, King NM, Kolli M, Mittal S, et al. Molecular Basis for Drug Resistance in HIV-1 Protease. Viruses. 2010;2:2509-35. 
[48] Huang L, Cao Y, Zhou J, Qin K, Zhu W, Zhu Y, et al. A conformational restriction in the influenza A virus neuraminidase binding site by R152 results in a combinational effect of I222T and H274Y on oseltamivir resistance. Antimicrob Agents Chemother. 2014;58:1639-45.

[49] van der Vries E, Veldhuis Kroeze EJ, Stittelaar KJ, Linster M, Van der Linden A, Schrauwen EJ, et al. Multidrug resistant 2009 A/H1N1 influenza clinical isolate with a neuraminidase I223R mutation retains its virulence and transmissibility in ferrets. PLoS Pathog. 2011;7:e1002276.

[50] van der Vries E, Collins PJ, Vachieri SG, Xiong X, Liu J, Walker PA, et al. H1N1 2009 pandemic influenza virus: resistance of the I223R neuraminidase mutant explained by kinetic and structural analysis. PLoS Pathog. 2012;8:e1002914.

[51] Abed Y, Pizzorno A, Bouhy X, Boivin G. Role of permissive neuraminidase mutations in influenza A/Brisbane/59/2007-like (H1N1) viruses. PLoS Pathog. 2012;7:e1002431.

[52] Butler J, Hooper KA, Petrie S, Lee R, Maurer-Stroh S, Reh L, et al. Estimating the fitness advantage conferred by permissive neuraminidase mutations in recent oseltamivir-resistant A(H1N1)pdm09 influenza viruses. PLoS Pathog. 2014;10:e1004065.

[53] Wagenaar TR, Ma L, Roscoe B, Park SM, Bolon DN, Green MR. Resistance to vemurafenib resulting from a novel mutation in the BRAFV600E kinase domain. Pigment Cell Melanoma Res. 2013;27:124-33. [54] Li MZ, Elledge SJ. Harnessing homologous recombination in vitro to generate recombinant DNA via SLIC. Nat Methods. 2007;4:251-6.

[55] Renzette N, Caffrey DR, Zeldovich KB, Liu P, Gallagher GR, Aiello D, et al. Evolution of the influenza A virus genome during development of oseltamivir resistance in vitro. J Virol. 2014;88:272-81.

[56] Hayden FG, Cote KM, Douglas RG, Jr. Plaque inhibition assay for drug susceptibility testing of influenza viruses. Antimicrob Agents Chemother. 1980;17:865-70.

[57] Edgar RC. MUSCLE: multiple sequence alignment with high accuracy and high throughput. Nucleic acids research. 2004;32:1792-7. 
[58] Marjuki H, Mishin VP, Chesnokov AP, De La Cruz JA, Davis CT, Villanueva JM, et al. Neuraminidase Mutations Conferring Resistance to Oseltamivir in Influenza A(H7N9) Viruses. J Virol. 2015;89:5419-26.

[59] Nguyen HT, Fry AM, Gubareva LV. Neuraminidase inhibitor resistance in influenza viruses and laboratory testing methods. Antivir Ther. 2012;17:159-73.

[60] Xu X, Zhu X, Dwek RA, Stevens J, Wilson IA. Structural characterization of the 1918 influenza virus H1N1 neuraminidase. J Virol. 2008;82:10493-501.

[61] Smith BJ, Colman PM, Von Itzstein M, Danylec B, Varghese JN. Analysis of inhibitor binding in influenza virus neuraminidase. Protein science : a publication of the Protein Society. 2001;10:689-96. 


\section{Tables}

Table 1. Amino acid regions of NA analyzed

\begin{tabular}{|lc|}
\hline Region & Amino acid positions \\
\hline Active-site proximal 1 & $112-121$ \\
\hline Active-site proximal 2 & $220-229$ \\
\hline Active-site proximal 3 & $271-280$ \\
\hline Active-site proximal 4 & $292-301$ \\
\hline Surface loop distant from active site & $83-92$ \\
\hline
\end{tabular}


Table 2. Experimental fitness effects of previously reported oseltamivir resistance mutants that were covered in the libraries analyzed in the study $[9,37,38,43,44,48$ $50,58,59]$

\begin{tabular}{|c|c|c|c|c|}
\hline Mutation & Subtype & No oseltamivir & $\begin{array}{c}1 \mu \mathrm{M} \\
\text { oseltamivir }\end{array}$ & $\begin{array}{l}\text { Increase in } \\
\text { fitness effect }\end{array}$ \\
\hline I117V & H5N1 & -0.14 & 0.12 & 0.26 \\
\hline E119V & H3N2, H7N9 & -1.10 & -1.00 & 0.10 \\
\hline I223K & H1N1, H7N9 & -0.54 & -0.15 & 0.39 \\
\hline I223R & H1N1, H7N9 & -0.56 & -0.13 & 0.43 \\
\hline I223M & H5N1 & -0.33 & 0.23 & 0.56 \\
\hline I223T & H1N1, H5N1 & -0.25 & -0.01 & 0.24 \\
\hline I223L & H5N1, H3N2 & -0.40 & -0.02 & 0.38 \\
\hline $\mathrm{H} 275 \mathrm{Y}$ & H1N1, H5N1 & -0.31 & 1.12 & 1.43 \\
\hline R293K & H3N2, H7N9 & -0.62 & -0.38 & 0.24 \\
\hline N295S & H1N1, H5N1, H3N2 & -0.30 & 0.49 & 0.79 \\
\hline
\end{tabular}


Figure 1

A

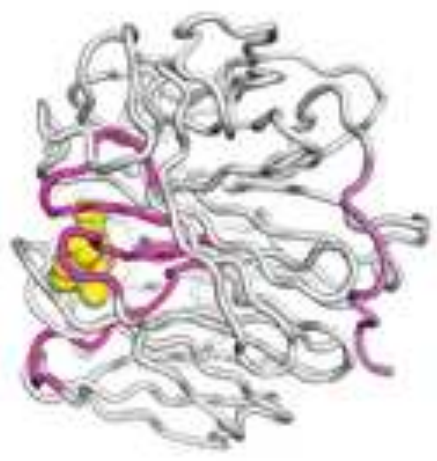

C

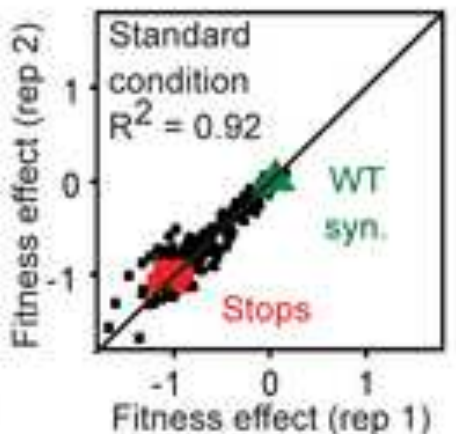

E
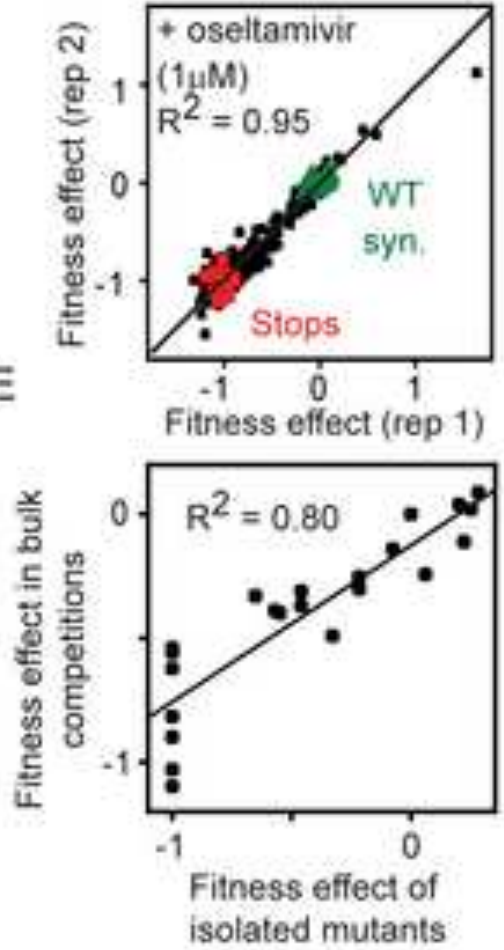

B

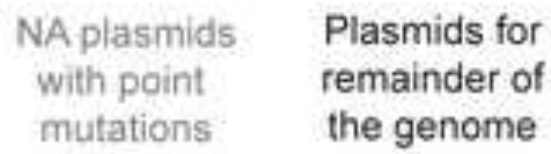

mutations the genome
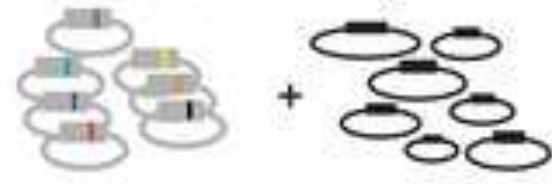

Co-transfection 293T/MDCK cell Collect supernatant

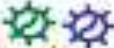

WO viral variants ख) ऐ
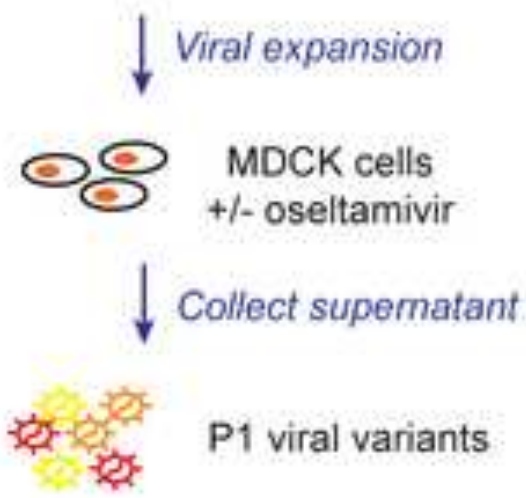

Deep sequence

Determine frequency change of each mutation from P0 to P1 
Figure 2

A

Active site proximal regions

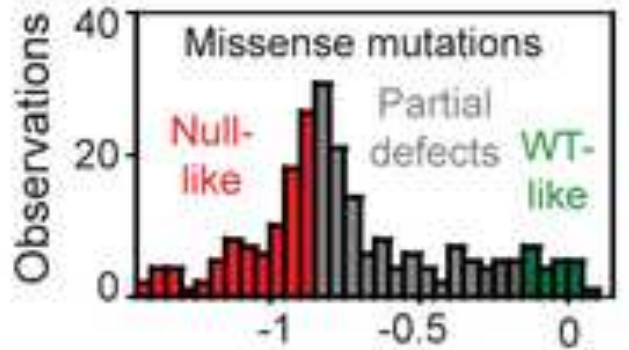

Fitness effect

C

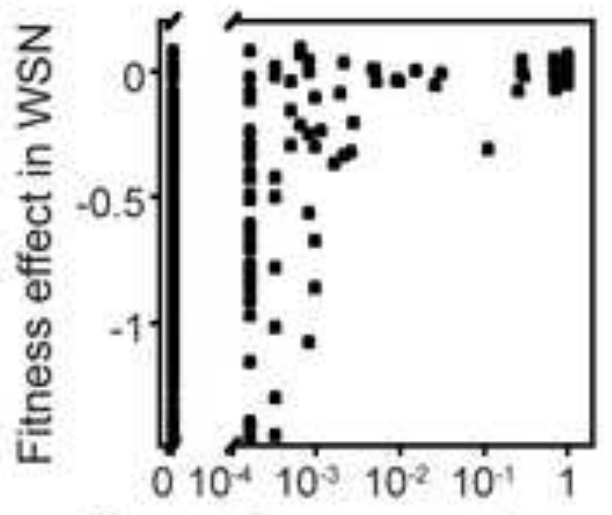

Mutant frequency observed in $\mathrm{H} 1 \mathrm{~N} 1$ natural isolates

E

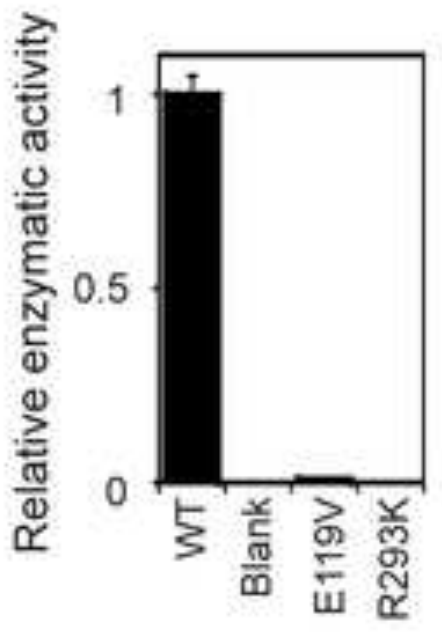

Substitution
B Exterior loop

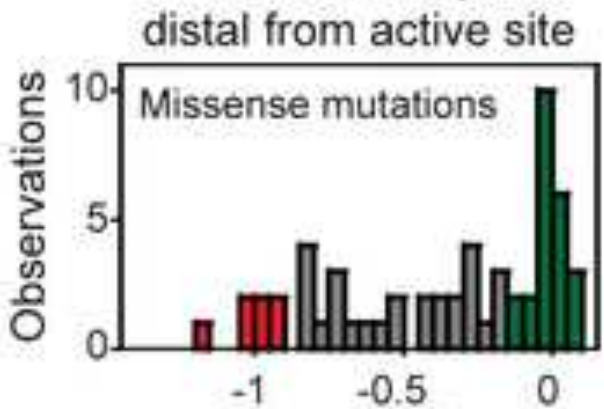

Fitness effect

D

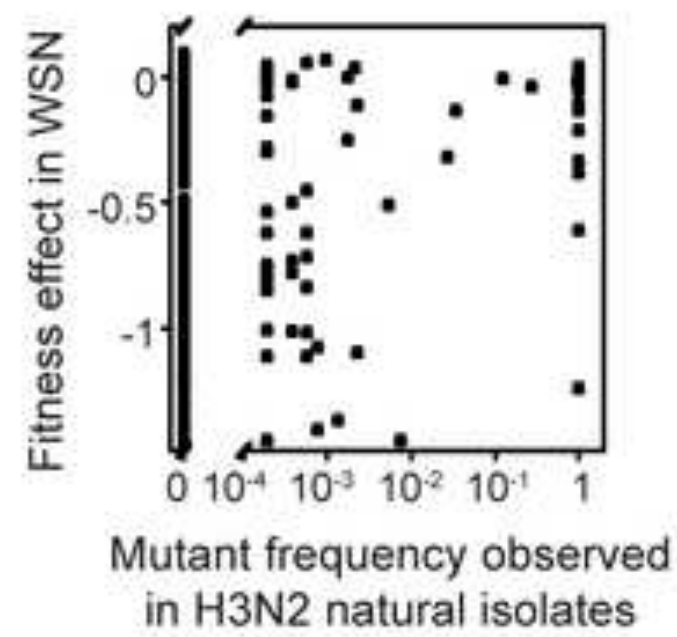


Figure 3

A

Fitness effect
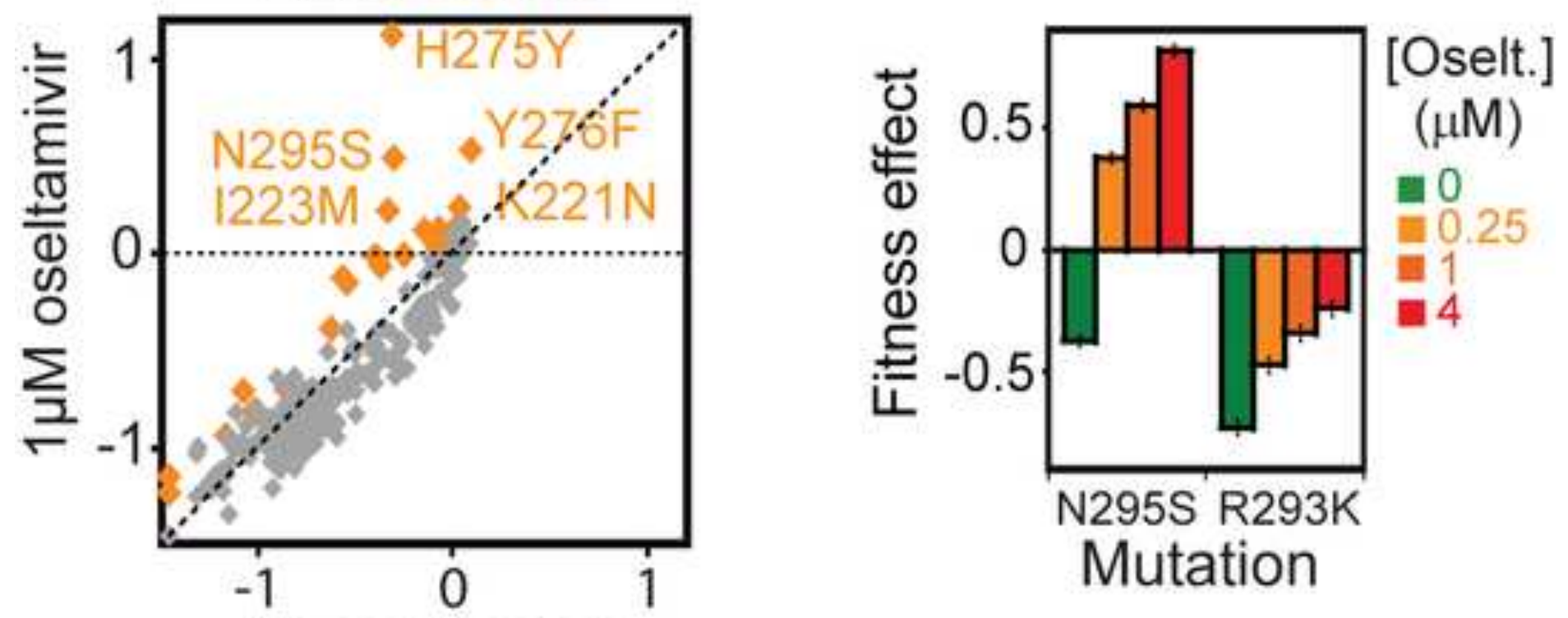

No oseltamivir

C

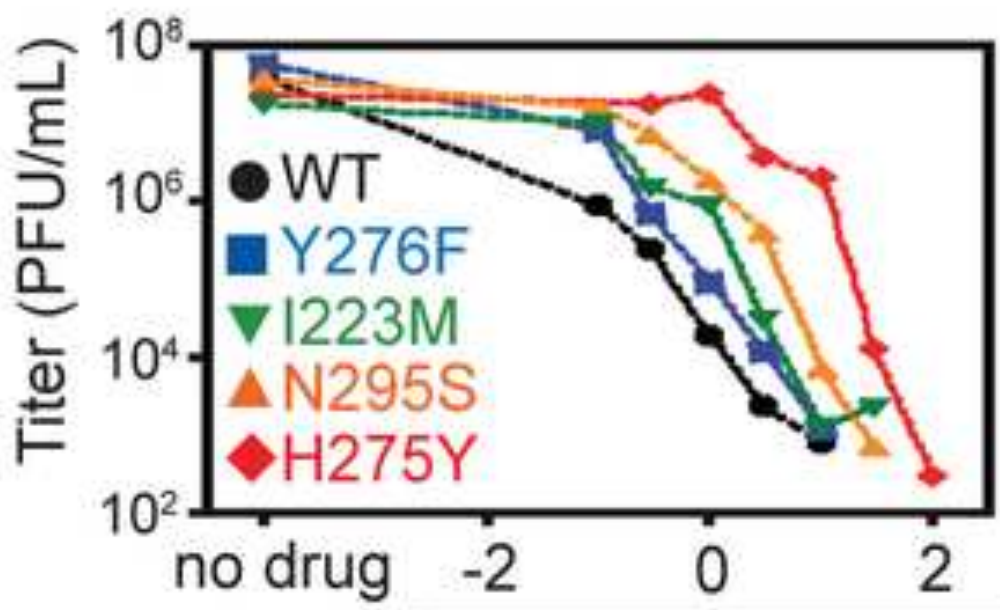

Log10 [Oseltamivir] $(\mu \mathrm{M})$ 
Figure 4

A

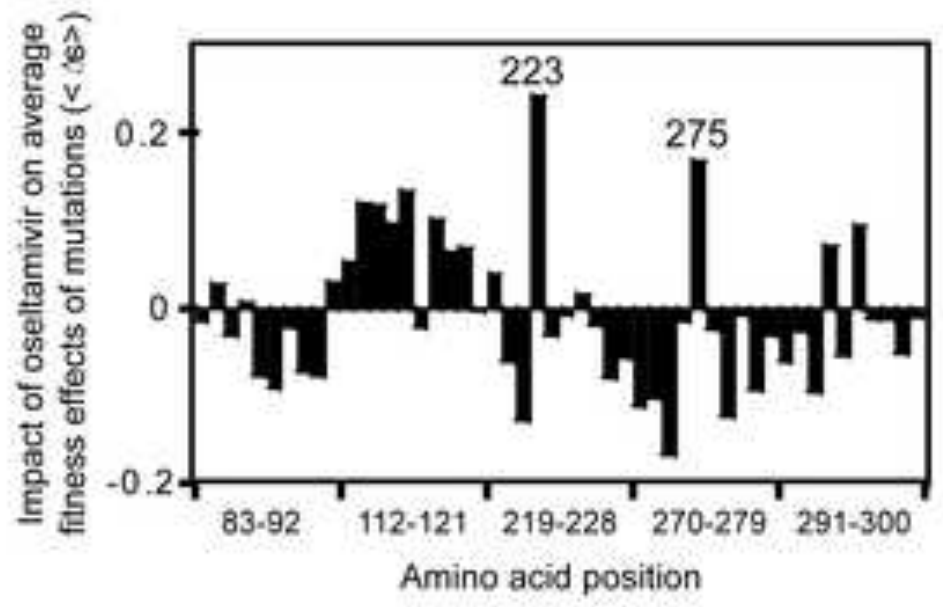

B

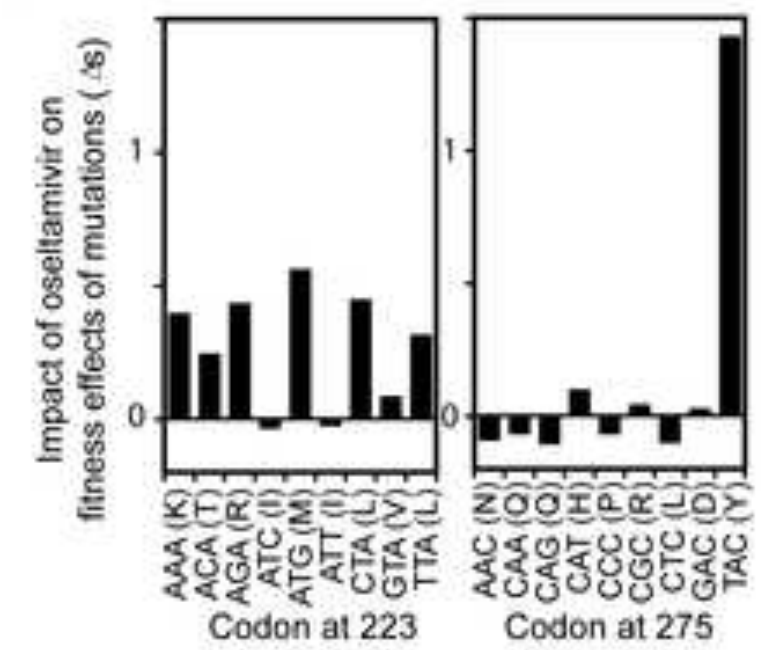

C

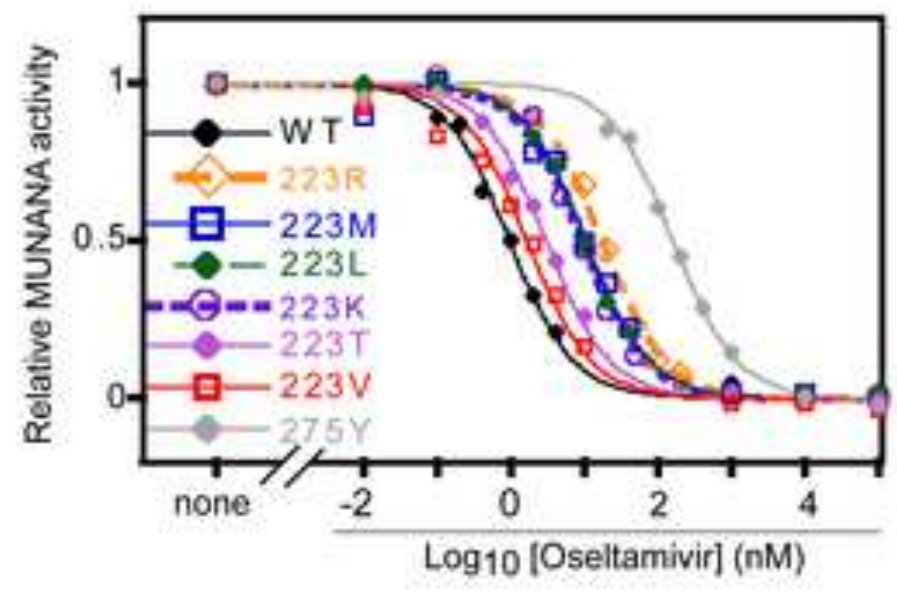

D

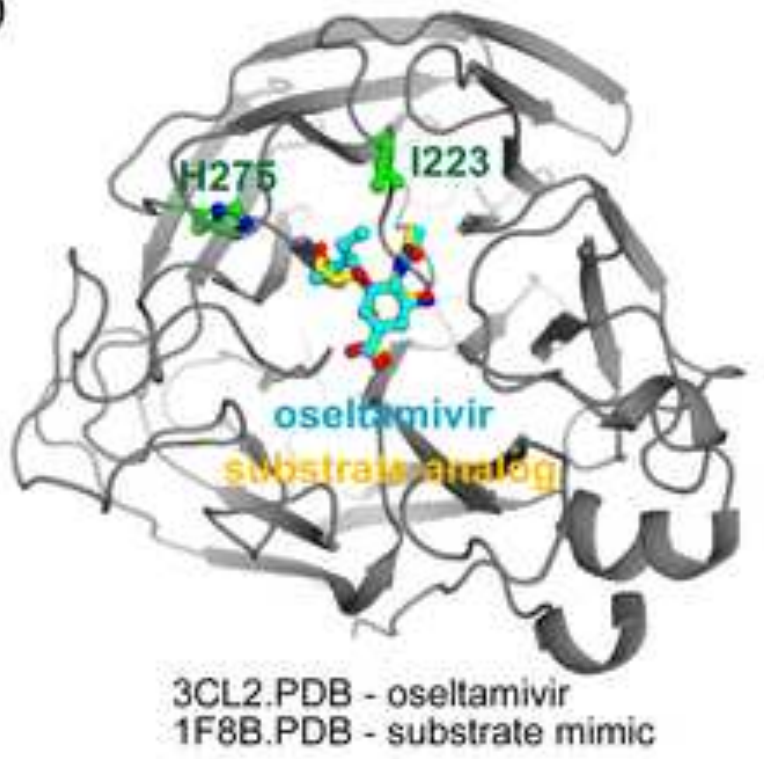


Figure 5

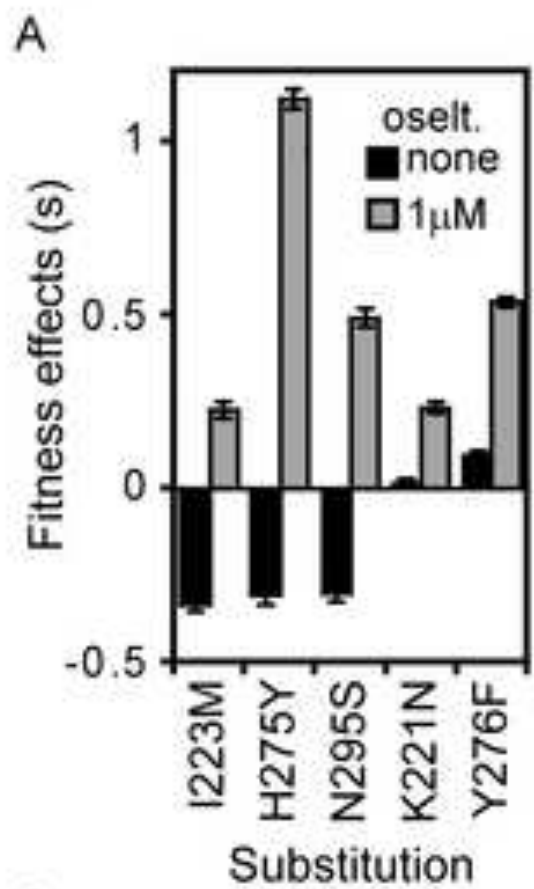

B
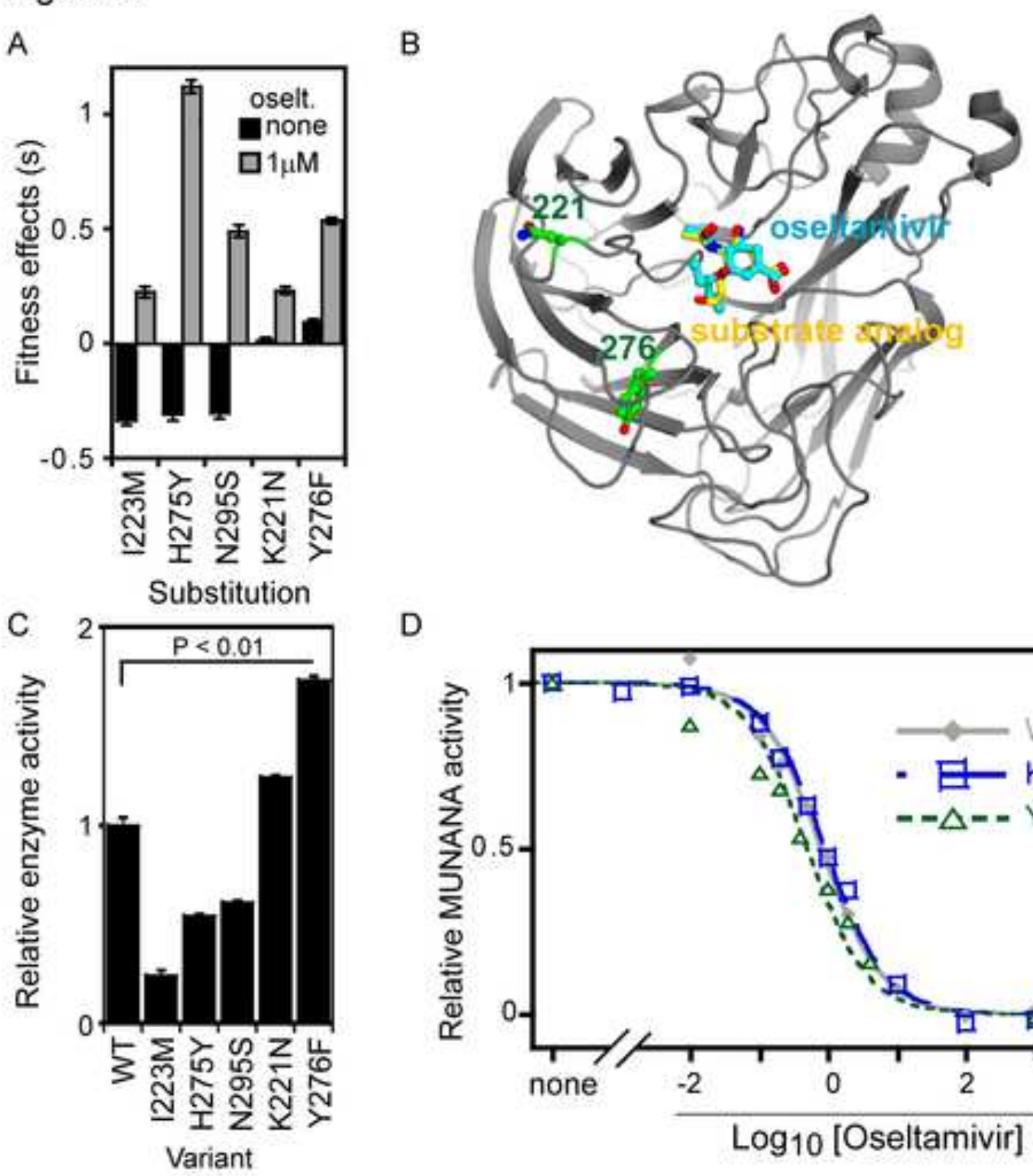

D

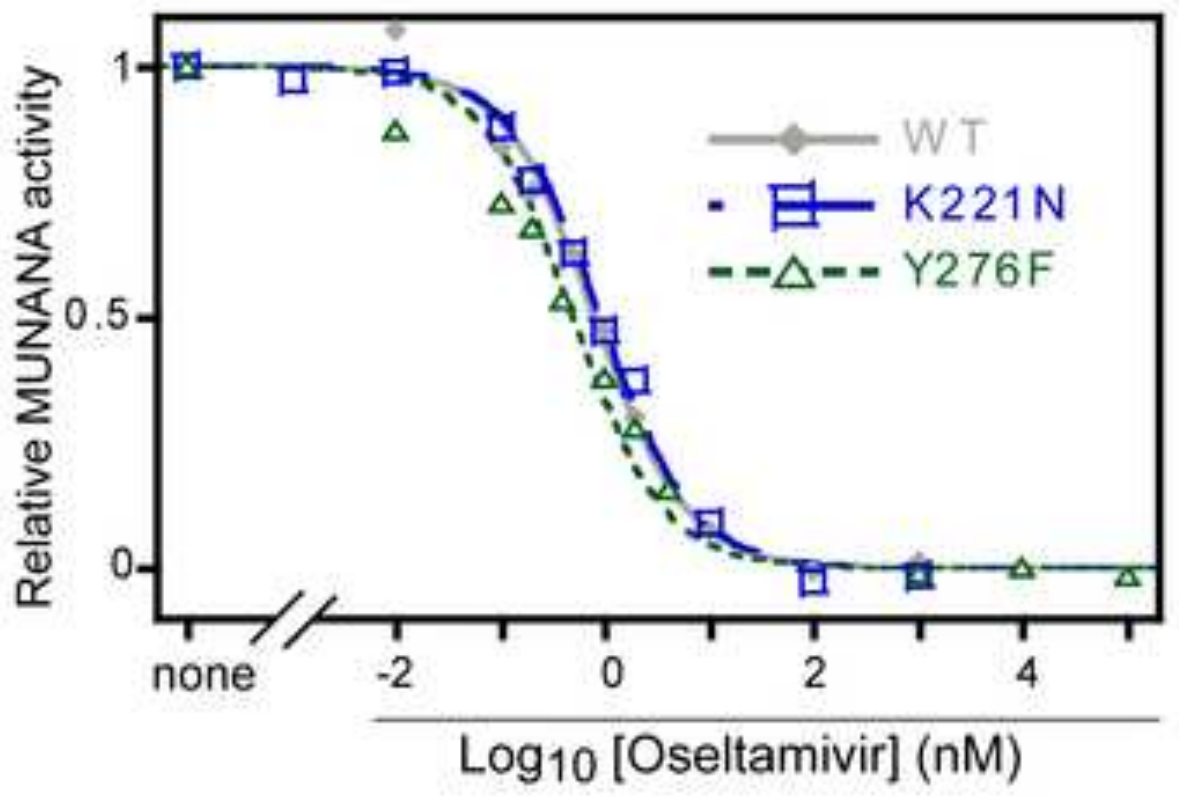


Figure 6
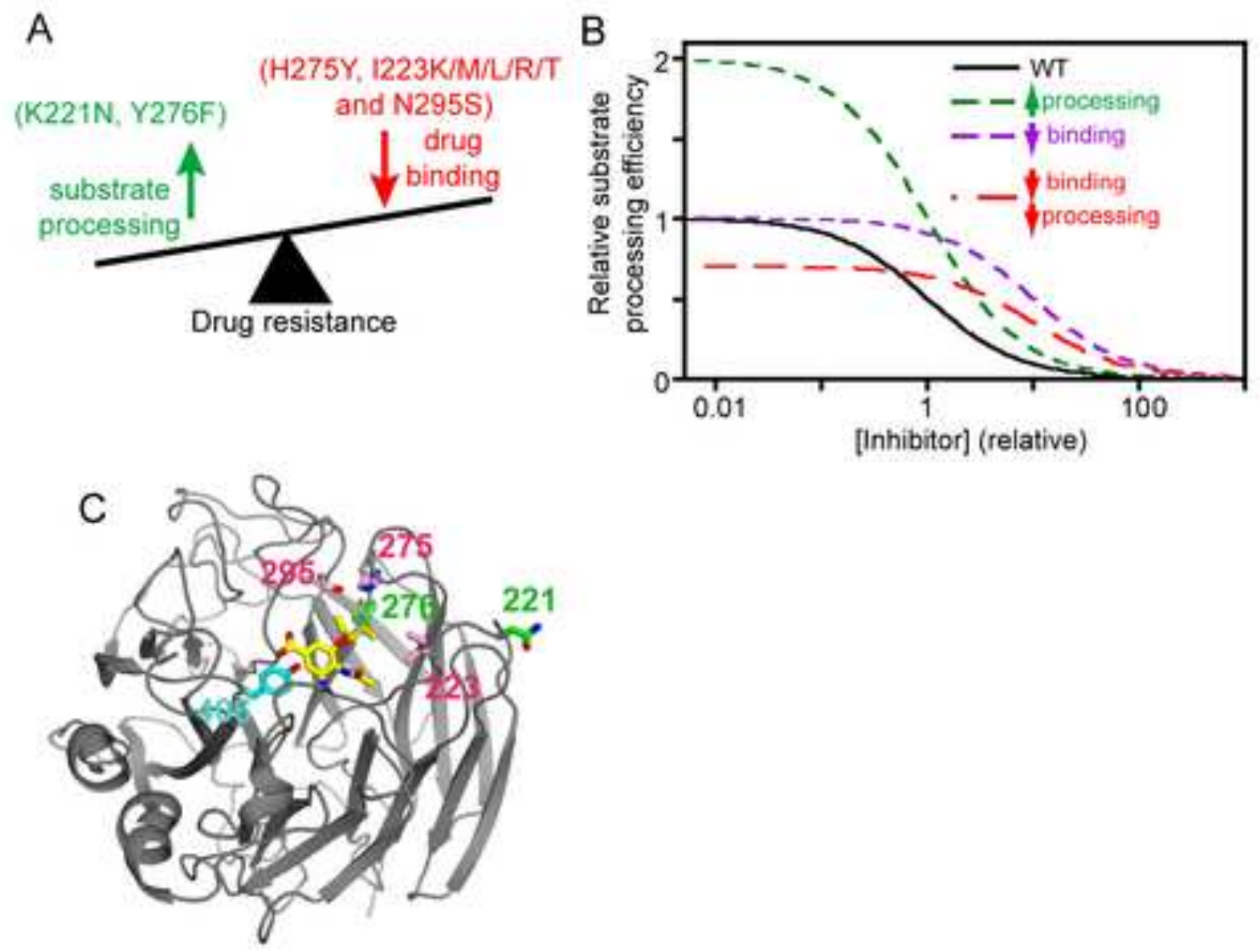

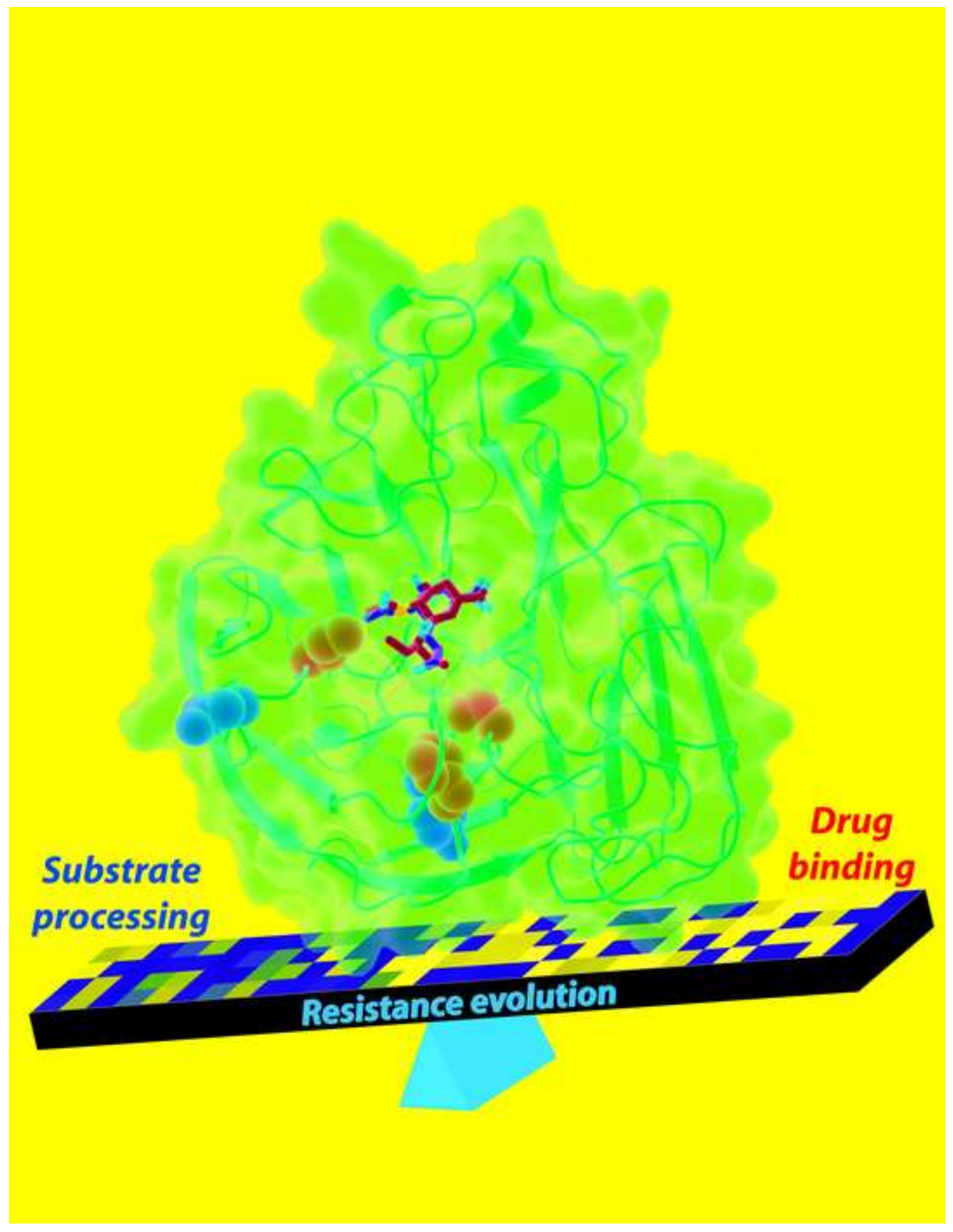

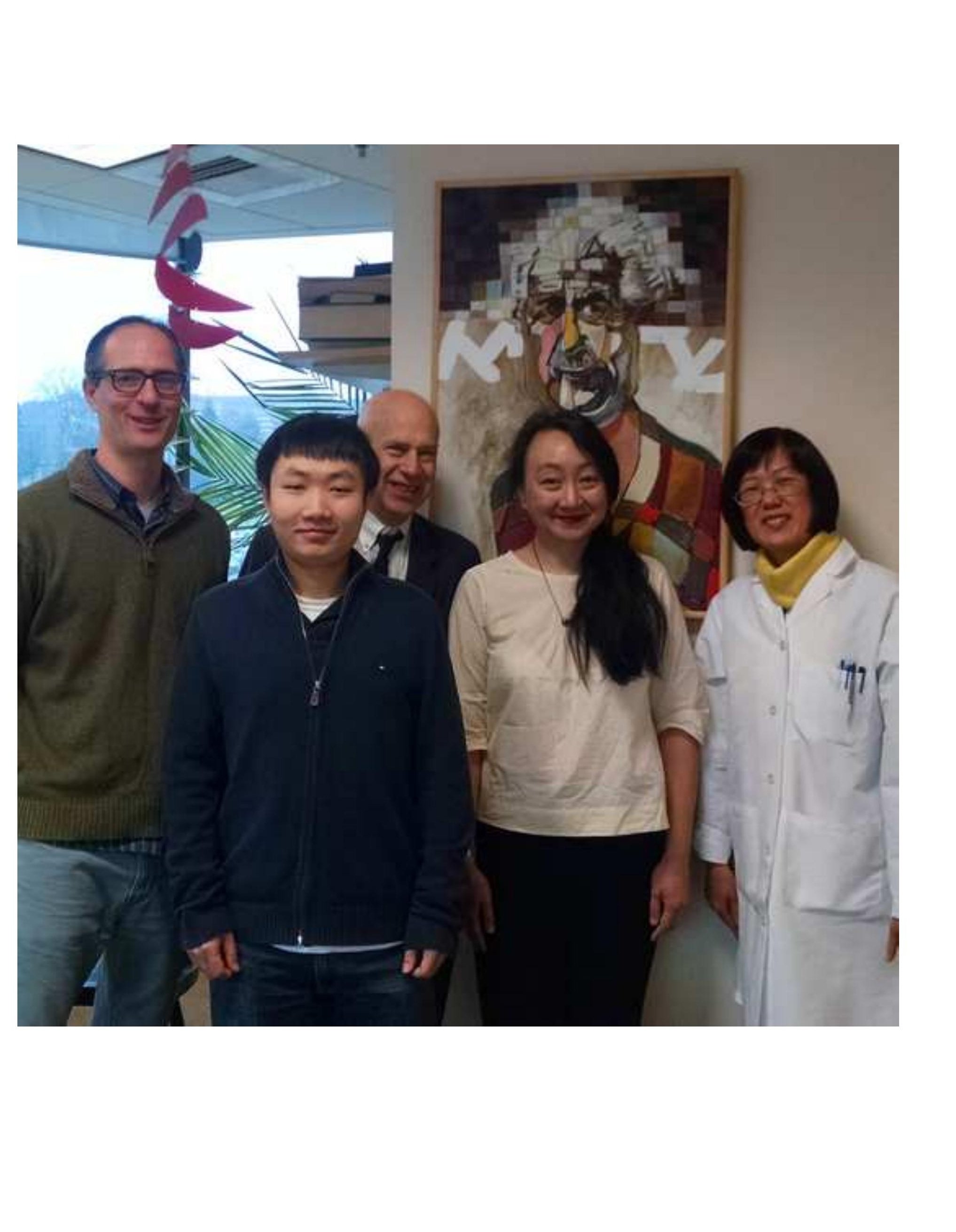